\title{
les concepts d'état limite et d'état critique et leurs applications pratiques à l'étude des argiles
}

\author{
par \\ F. Tavenas \\ Professeur agrégé \\ Département de Génie civil \\ Université Laval, Québec, Canada
}

et

\section{S. Leroueil}

Ingénieur

Terratech Limitée

Montréal, Canada

Depuis une dizaine d'années, le groupe de recherche en géotechnique de l'Université Laval poursuit un vaste programme d'étude des argiles molles et sensibles, axé sur la détermination des propriétés in situ de ces argiles et de leur comportement comme fondation de remblais. Ce programme a été établi de manière à fournir une vue globale du problème et il a comporté:

- l'étude des différentes techniques d'essai in situ et de prélèvement non remanié des argiles sensibles;

- des programmes expérimentaux en laboratoire visant à la détermination des caractéristiques de résistance, de déformation et de fluage des argiles à l'état intact et remanié ;

- la construction et l'observation de quatre remblais expérimentaux à Saint-Alban, Québec :

- l'accumulation de données sur le comportement de nombreux autres remblais sur argiles molles, tant au Québec quailleurs dans le monde :

- le développement d'une coopération étroite avec le département des sols et fondations du LCPC, Paris, sur le thème du comportement des remblais sur sols compressibles. Cette coopération, établie dans le cadre des accords Franco-Québécois de Coopération Scientifique, a permis une mise en commun des expériences, des données et des idées et a fourni un vaste champ d'application et de vérification de nos idées respectives.

Une telle approche globale est certes une des conditions essentielles à un progrès rapide de la recherche, mais elle ne peut vraiment être efficace que dans la mesure où elle repose sur un appareil théorique convenable. Or il s'est rapidement avéré que l'approche classique de la mécanique des argiles ne pouvait constituer une base théorique adéquate. En effet les divisions très nettes entre les problèmes de résistance et de déformation, de fluage et de consolidation, de comportement drainé et non drainé, rendent très difficile la compréhension des comportements naturels qui intègrent généralement l'ensemble de ces problèmes. Au contraire, nous avons pu établir que les concepts d'état limite et d'état critique proposés par Roscoe, Schofield et Wroth [18] s'appliquent bien aux argiles naturelles et peuvent être élargis de façon à en décrire, sous une forme intégrée, toutes les propriétés rhéologiques.
Après un bref rappel des principes de base d'état limite et d'état critique, le but de cet article est de montrer succinctement :

- comment la notion d'état limite représente effectivement le comportement d'une argile naturelle dans tous ses aspects :

- comment cette notion peut être utilisée de manière simple pour aider à l'interprétation des essais in situ et en laboratoire :

- comment surtout, en imposant l'étude précise des conditions de contraintes effectives, elle conduit à une approche toute nouvelle de l'étude des ouvrages sur sol compressible.

Cet article étant une synthèse de travaux récents, le lecteur est renvoyé aux différentes publications citées en référence pour obtenir une vue détaillée des différents problèmes abordés ici.

1 Rappel des principes de base des états limite et critique

Les concepts d'état limite et d'état critique ont été mis en forme entre 1958 et 1968 par Roscoe et ses collègues à Cambridge $[18,19,20]$. Dans leur forme originale, ils ne s'appliquent qu'à des argiles normalement consolidées, isotropes, donc à des matériaux de nature fictive; mais nous allons voir que tous les principes qui forment la base de ces concepts sont qualitativement applicables aux argiles naturelles, surconsolidées et anisotropes.

$\mathrm{Si}$ on soumet une argile, remaniée à une teneur en eau proche de sa limite de liquidité, à une consolidation isotrope, son indice des vides va diminuer suivant la loi bien connue

$$
\Delta e=C_{c} \Delta \log p^{\prime}
$$

représentée par la droite $O E$ (fig. 1). 


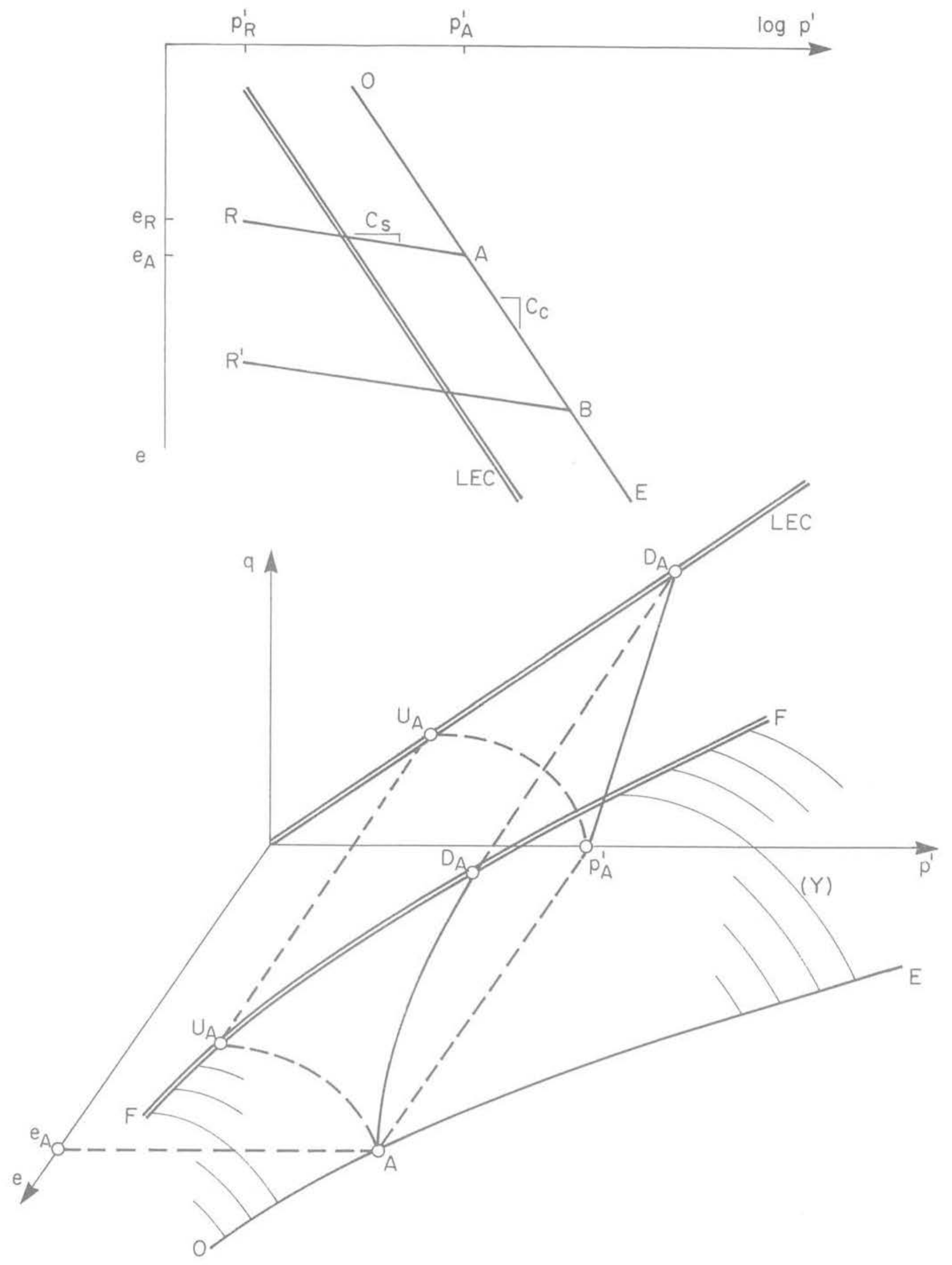

Fig. 1 Principes d'état limite et d'état critique 
Si, après avoir atteint la condition A (fig. 1) on réduit la contrainte effective moyenne de $p_{A}$ à $p_{\dot{A}}$, l'indice des vides du spécimen étudié va augmenter légèrement de $e_{A} a ̀ e_{R}$. suivant une loi du type

$$
\Delta \mathrm{e}=\mathrm{C}_{\mathrm{s}} \Delta \log \mathrm{p}^{\prime}
$$

où $\mathrm{C}_{\mathrm{S}}$ est nettement inférieur à $\mathrm{C}_{\mathrm{c}}$. Si on reprend alors le chargement isotrope la variation $\mathrm{e}=f\left(p^{\prime}\right)$ va d'abord suivre RA, puis, pour des contraintes effectives moyennes supérieures à $p_{A}^{\prime}$, retrouver la loi suivie au début de l'essai, c'est-à-dire OE.

Si, à partir d'un état suivant, $B$, on procède à un nouveau cycle de déchargement-rechargement, la loi $e=f\left(p^{\prime}\right)$ sera telle que BR'BE.

On peut donc définir pour le spécimen étudié deux types de comportement effort-déformation :

- entre R et A ou $R^{\prime}$ et $B$, les déformations sont petites et essentiellement réversibles et peuvent être approchées par une loi élastique. De manière plus générale tout le demi espace e-log $p^{\prime}$ à gauche de OE, qui correspond au domaine surconsolidé, est un domaine de comportement de type élastique :

- entre A et B les déformations sont grandes et essentiellement irréversibles, et donc de nature plastique, La droite $\mathrm{OE}$, qui correspond à l'état normalement consolidé de l'argile représente également un domaine de comportement plastique :

- les points A et B constituent des états limites entre comportement élastique et comportement plastique et la droite OE est aussi la trace dans le plan e $-\log p^{\prime}$ du lieu de ces états limites; on l'appelle droite d'état limite.

Si maintenant on réalise des essais de cisaillement triaxial drainé et non drainé à partir d'un point $\mathrm{A}$ quelconque de la droite d'état limite, Roscoe et al [18] ont montré que les chemins d'état suivis dans l'espace e, p', q (fig. 1) étaient situés sur une surface unique pour une argile donnée. Cette surface, dont OE est la trace dans le plan e, p', est appelée surface d'état limite (SEL). Elle représente également le lieu des états normalement consolidés de l'argile étudiée. Lorsqu'on poursuit les essais de cisaillement triaxial jusqu'à grandes déformations, Roscoe et al [18] ont montré qu'on finit par atteindre en $U_{A}$ ou $D_{A}$ des états stables tels que :

$$
\frac{\partial p}{\partial \epsilon}=\frac{\partial q}{\partial \epsilon}=0
$$

et

$$
\frac{\partial u}{\partial \epsilon} \text { ou } \frac{\partial e}{\partial \epsilon}=0
$$

Ces états, appelés états critiques, sont situés sur une ligne FF qui constitue une borne supérieure de la SEL, et dont la projection dans le plan $\mathrm{p}^{\prime}$. q est une droite de pente

$$
M=\frac{6 \sin \varphi^{\prime}}{3-\sin \varphi^{\prime}} \simeq \frac{\varphi^{\prime(0)}}{25}
$$

qui correspond en fait à la droite de Mohr-Coulomb. Cette droite est appelée ligne d'état critique (LEC). Dans le plan $e-\log p^{\prime}$, la LEC se projète sous la forme d'une droite de pente $C_{c}$, parallèle à la ligne d'état limite $O E$. Ceci implique que, dans des essais non drainés sur une argile donnée, il existe un rapport constant entre le déviateur à l'état critique et la pression isotrope de consolidation initiale; on retrouve là, dans une certaine mesure, le principe du rapport $c_{u} / \sigma_{0}^{\prime}$ caractéristique de chaque argile établi de manière empirique par Skempton [24] puis Bjerrum [3]. Ainsi donc, la connaissance de la SEL et de la LEC d'une argile permet d'en décrire complètement le comportement. Tout état e, p', q situé à l'intérieur de l'espace délimité par la SEL et la LEC correspond à un comportement de type élastique, décrit en première approximation par un module volumétrique $\mathrm{K}$ ou le coefficient $\mathrm{C}_{\mathrm{S}}$ et un module de cisaillement $\mathrm{G}$ (ou $E_{u}$ ). Tout état de contrainte situé sur la SEL amène l'argile en état normalement consolidé avec des grandes déformations calculables, à partir de $\mathrm{C}_{\mathrm{c}}$ pour les déformations volumétriques, et à partir d'une loi d'écoulement plastique pour les déformations de cisaillement. Tout état de contrainte situé sur la LEC, définie par la connaissance de l'angle de frottement effectif $\varphi^{\prime}$, provoque la rupture de l'argile. Par conséquent, le comportement d'une argile est défini en première approximation par la connaissance des paramètres suivants:

- l'indice des vides $e_{0}$ :

- les paramètres de déformation dans le domaine surconsolidé, soit $\mathrm{C}_{\mathrm{S}}$ ou $\mathrm{K}$ et $\mathrm{G}$ :

- I'indice de compressibilité de l'argile normalement consolidée $\mathrm{C}_{\mathrm{c}}$;

- l'angle de frottement effectif $\varphi^{\prime}$;

- l'équation $(Y)$ de la surface d'état limite.

Les premiers paramètres sont classiques et relativement simples à déterminer. On notera cependant qu'ils correspondent à une description simplifiée du comportement d'une argile et qu'ils devront être complétés ou modifiés pour tenir compte des propriétés d'anisotropie, de non linéarité et de fluage des argiles naturelles.

Pour ce qui est de l'équation $(Y)$ de la SEL, Roscoe et al $[19,20]$ ont cherché à développer une approche mathématique à partir de la théorie de plasticité. II en a résulté les modèles Cam-Clay et Cam-Clay modifié applicables strictement aux argiles isotropes. Ohta et Hata [16] ont par ailleurs modifié le modèle Cam-Clay pour tenir compte dans une certaine mesure de l'anisotropie. II reste que ces modèles sont difficilement applicables tels quels aux argiles naturelles.

On peut au contraire utiliser une approche d'abord expérimentale, consistant à déterminer la forme des SEL de différentes argiles naturelles à partir d'essais de laboratoire, et à préciser la nature physique des phénomènes d'état limite pour ensuite, éventuellement, en développer une formulation mathématique.

\section{2 États limites des argiles naturelles}

La détermination de la SEL d'une argile naturelle se fait par essais triaxiaux sur des échantillons non remaniés. Deux techniques peuvent être utilisées.

Crooks et Graham (5), travaillant sur une série d'échantillons provenant d'une profondeur donnée, à laquelle correspondent des valeurs communes de $e_{0}, \sigma_{\text {vo }}$ et $\sigma_{p}^{*}$, reconsolident d'abord chaque échantillon sous les contraintes en place $\sigma_{\text {vo }}$. $K_{0} \sigma_{\text {vo }}^{*}$. Ils appliquent ensuite à chaque échantillon un chargement triaxial drainé suivant un cheminement de contrainte tel que $1,2, \ldots 7$ (fig. 2a) en mesurant la variation de volume de l'échantillon en fonction des contraintes appliquées. Par définition, l'état limite le long de chacun de ces cheminements est l'état de contrainte qui correspond à l'initiation des grandes déformations irréversibles. L'ensemble de ces états limites définit une courbe gauche sur la surface d'état limite, dont on représente la projection dans le plan p', q (fig. 2a). En faisant la même série d'essais sur des échantillons provenant de plusieurs profondeurs dans le même dépôt on obtient plusieurs lignes de la SEL et finalement une image suffisante de l'ensemble de cette surface. Cette technique a l'avantage de bien reproduire les conditions de contrainte in situ. Elle présente par contre deux difficultés: d'une part les chemi- 


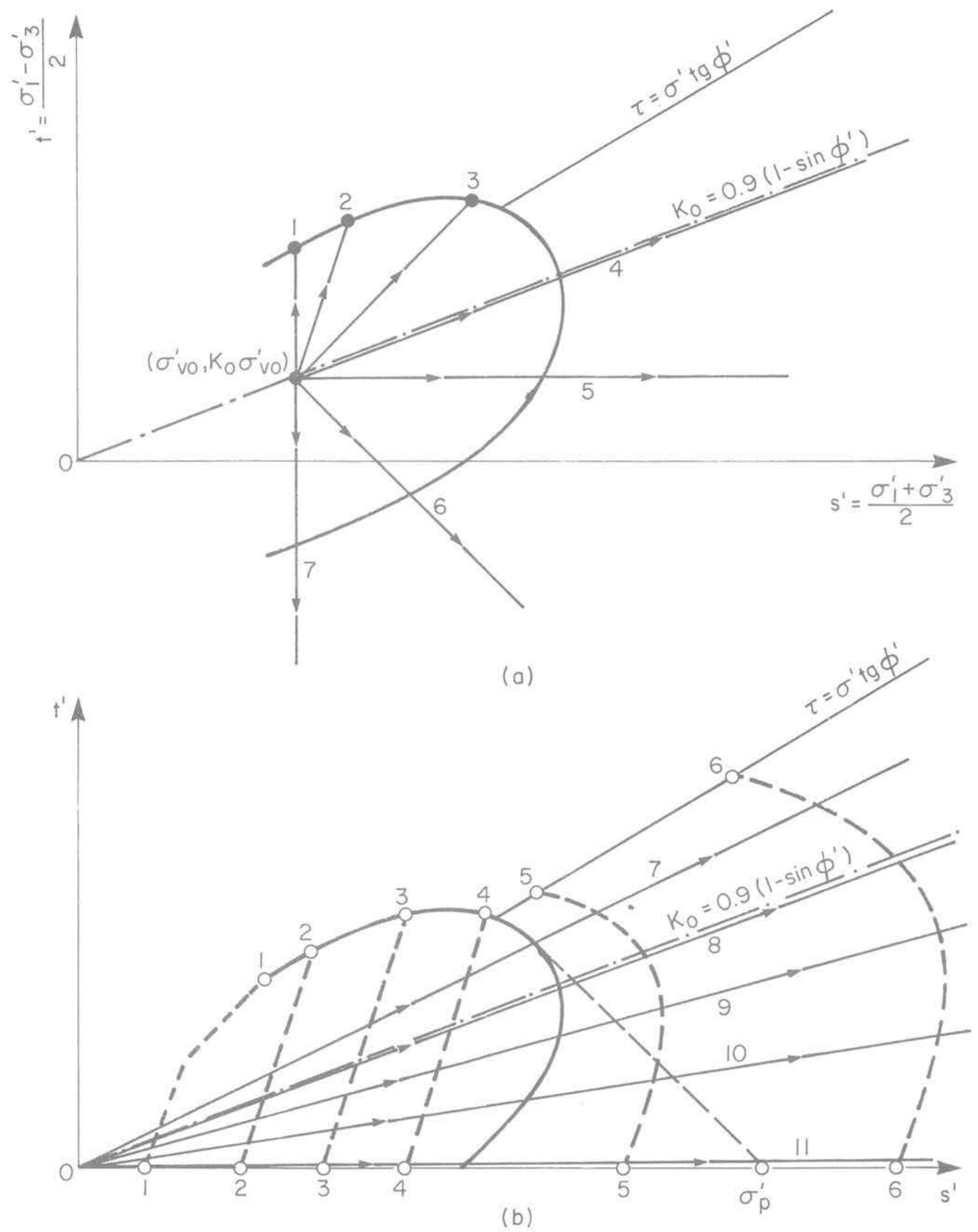

Fig. 2 Cheminements des contraintes effectives dans les essais servant à la définition de la SEL 
nements 6 et 7 ne peuvent être obtenus qu'avec des équipements triaxiaux spéciaux ; d'autre part, les variations de volume étant faibles ou nulles suivant les cheminements 1 et 7 , il faut utiliser une autre composante du tenseur des déformations pour définir l'état limite, ce qui peut conduire à des erreurs $(5,9)$.

Tavenas et Leroueil (27) ont suggéré l'utilisation d'une autre technique, qui ne fait appel qu'à des essais classiques. Sur une série d'échantillons provenant d'une profondeur donnée on réalise d'abord un essai œdométrique pour déterminer la pression de préconsolidation $\sigma_{\mathrm{p}}^{\prime}$ puis une série d'essais triaxiaux CIU (consolidés isotropiquement non drainés) à partir de pressions de consolidation de l'ordre de $0.1,0.25,0.35,0.5 \sigma_{\dot{p}}^{\prime}$ (cheminements 1, 2, 3, 4 . fig. 2b). L'expérience montre que lors de ces essais sur l'argile surconsolidée la première ruptưre, correspondant au pic de la courbe $\tau-\epsilon$, correspond à un état limite. On peut, pour vérification, réaliser des essais CID (consolidés isotropiquement drainés) à partir de pressions de l'ordre de 0.1 à $0.2 \sigma_{p}^{\prime}$; les variations de volume en fonction des contraintes confirment que la rupture est bien un état limite. On réalise par ailleurs une série d'essais de consolidation triaxiale anisotrope à rapport de contrainte constant (cheminements 7 à 11) dans lesquels on observe les variations de volume en fonction des paliers de contrainte appliqués. Enfin deux essais triaxiaux $\mathrm{CIU}$ à forte pression de consolidation $\left(0.8\right.$ et 1.2 ou $1.5 \sigma_{p}^{\prime}$, cheminements 5 et 6 , fig. 2 b) permettent la détermination de l'angle de frottement effectif $\varphi^{\prime}$ de l'argile normalement consolidée et donc de LEC.

La seule difficulté reliée à la détermination de la SEL d'une argile naturelle provient de la nécessité de disposer d'une série de 7 à 12 spécimens intacts provenant de la même profondeur et ayant les mêmes valeurs de $e_{0}, \sigma_{\text {vo }}^{*}$ et $\sigma_{p}^{*}$. Le prélèvement de blocs en fond de fouille ne peut en général être envisagé pour des raisons économiques mais l'utilisation de gros carottiers tels que l'appareil $\varnothing 200 \mathrm{~mm}$ développé à I'Université Laval [22] conduit à des résultats excellents en ce qui concerne tant la qualité que le coût des échantillons. On peut bien sûr utiliser des échantillons de plus petit diamètre provenant de trous voisins mais il faut alors accepter d'une part un certain remaniement, d'autre part une dispersion des valeurs de $e_{0}, \sigma_{\text {vo }}$ et $\sigma_{p}^{\prime}$ et partant des états limites obtenus.

La figure 3 présente les états limites obtenus par les deux techniques décrites ci-dessus, sur des échantillons prélevés à $3.0 \mathrm{~m}$ de profondeur dans le dépôt d'argile sensible de Saint-Alban (Quebec). Le détail de ces essais et des résultats est présenté par Tavenas et Leroueil [27] et par Tavenas et al [30]. On notera tout d'abord la dispersion assez faible des résultats, due à la qualité des échantillons prélevés en $\varnothing 200 \mathrm{~mm}$ et à l'homogénéité du dépôt de Saint-Alban. Quant à la coupe de la SEL correspondant aux valeurs locales de $e_{0}$ et $\sigma_{p}$ elle présente une forme approximativement elliptique, plus ou moins centrée sur la droite $K_{0}$ et elle recoupe cette droite $K_{0}$ et elle recoupe cette droite $K_{0}$ à une valeur de $\sigma_{j}^{\prime}=\sigma_{\mathrm{p}}^{\prime}$. Si on compare ce résultat à la solution théorique correspondant au modèle Cam-Clay modifié (Roscoe et Burland, [20], on retrouve dans les deux cas une forme elliptique, mais l'état de contrainte anisotrope, $\mathrm{K}_{0}$, sous lequel l'argile naturelle a été déposée et consolidée conduit à un déplacement de l'axe de symétrie de la SEL, de la ligne $\mathrm{t}^{\prime}=0$ dans le cas du modèle Cam-Clay applicable à l'argile isotrope, à la ligne $\mathrm{K}_{0}$ dans le cas de l'argile naturelle. Une forme semblable a été observée dans d'autres argiles (fig. 4) et elle semble en fait universelle (Leroueil et Tavenas, [9]).

Fig. 3 Coupe de la SEL de l'argile de Saint-Alban à $3.0 \mathrm{~m}$ de profondeur

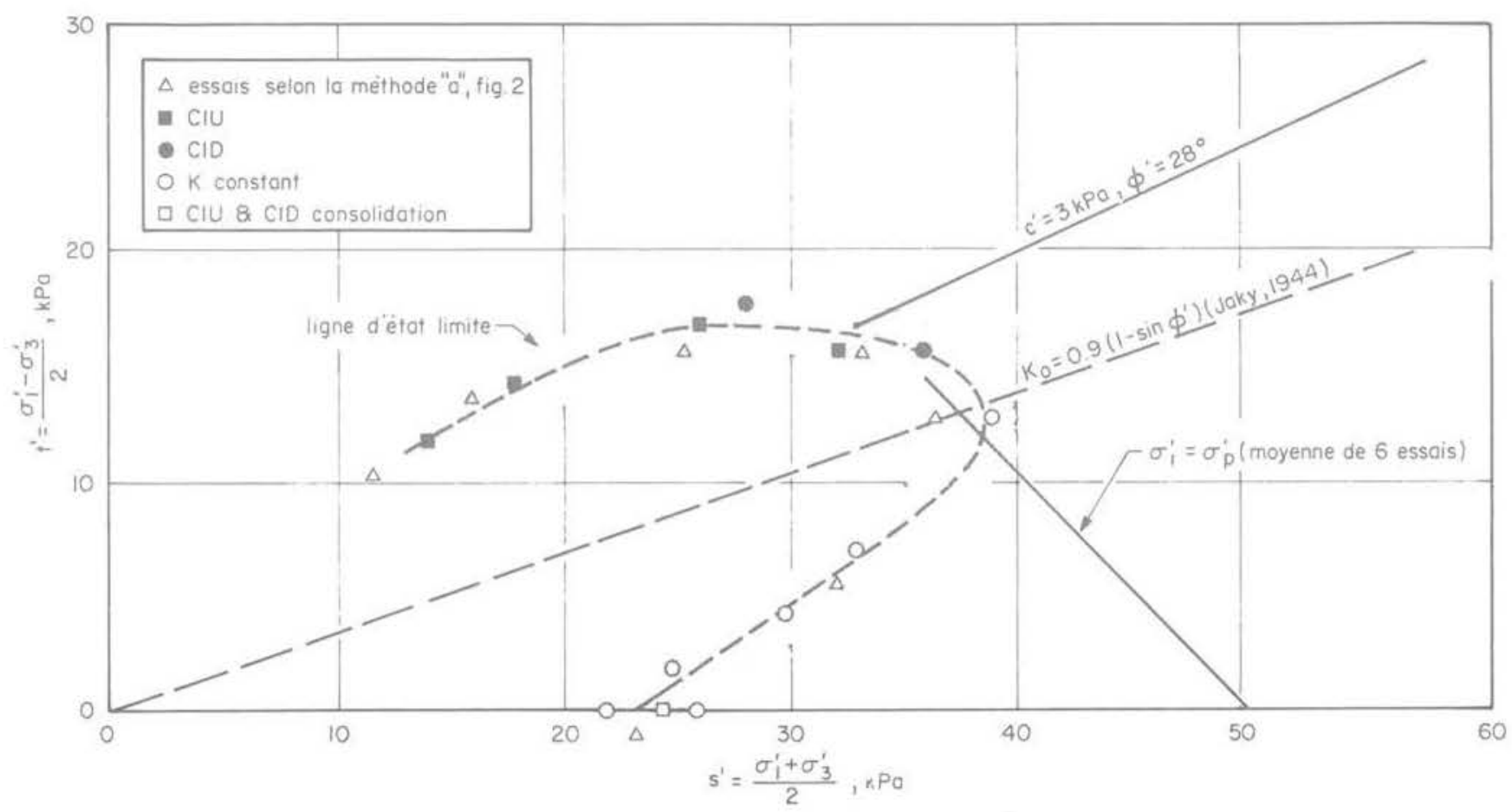



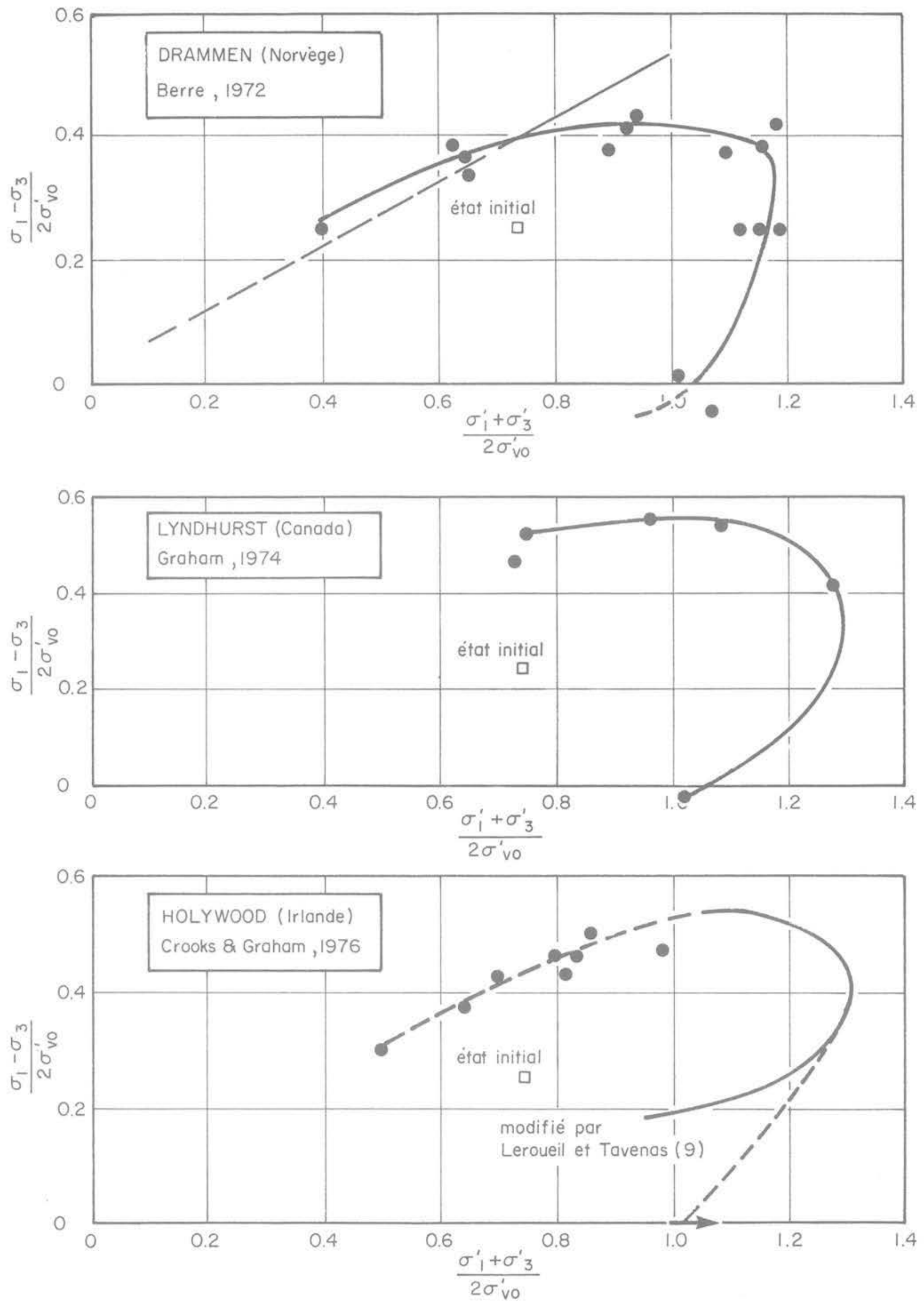

Fig. 4 SEL d'argiles de différentes origines (d'après Leroueil, [8]) 


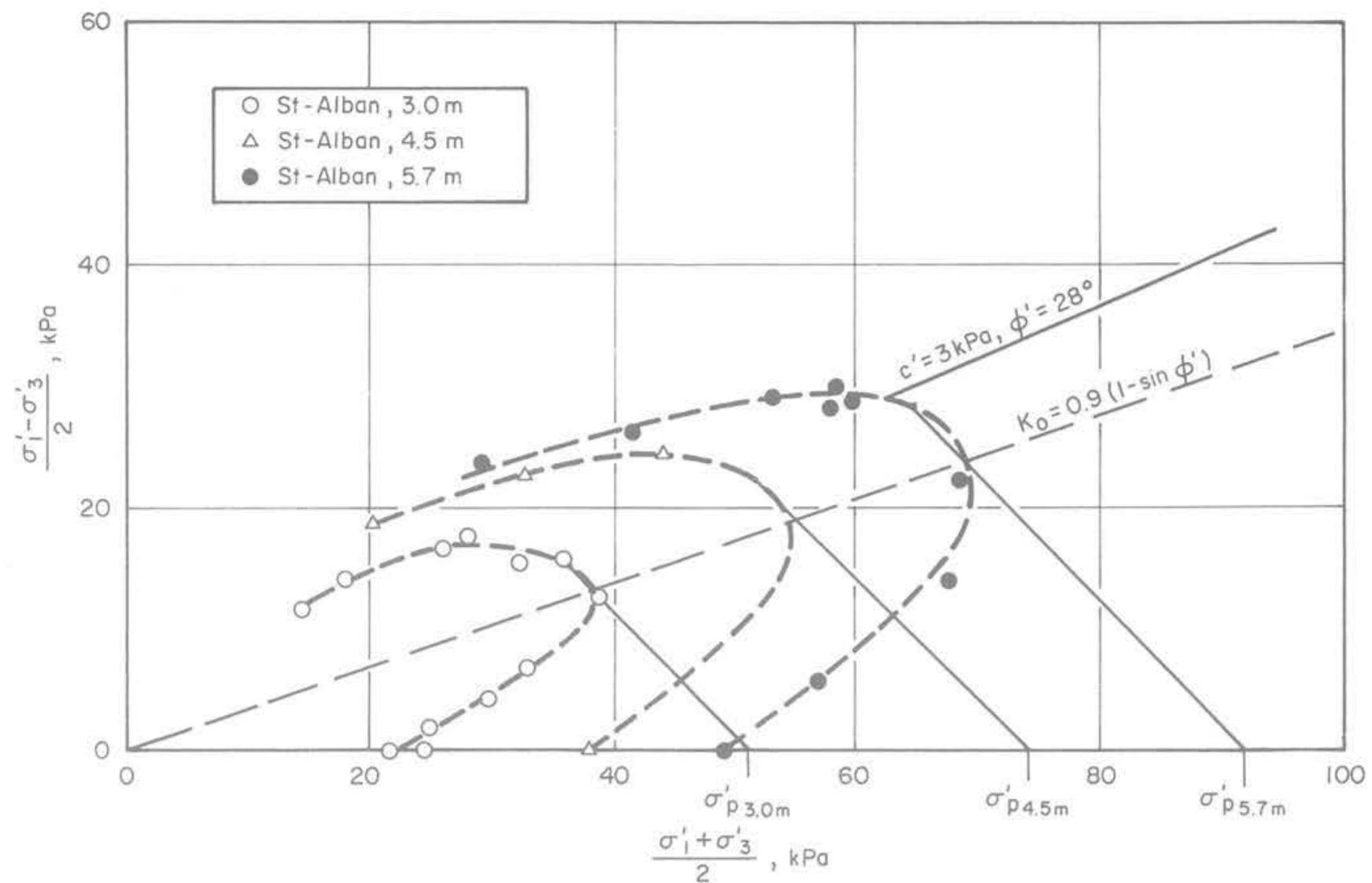

Fig. 5 Coupes de la SEL de l'argile de Saint-Alban à différentes profondeurs (d'après Leroueil, [8] et Trak [32])

La figure 5 présente les états limites obtenus en faisant des essais similaires sur des échantillons prélevés à différentes profondeurs dans le dépôt de Saint Alban. Les coupes de la SEL correspondant à chaque profondeur et donc à chaque valeur de $e_{o}$ et $\sigma_{p}^{\prime}$ sont bien toutes de forme elliptique centrée sur la ligne $K_{0}$ et elles sont localisées le long de cette ligne en fonction de la valeur locale de $\sigma_{\mathrm{p}}^{\prime}$. On peut donc en conclure que la forme et la position des coupes de la SEL dans des plans $\mathrm{e} \approx$ Cste dans l'espace e, $\mathrm{p}^{\prime}$, $\mathrm{q}$, sont fixées par l'état de contrainte maximum ayant régné en cours de formation du dépôt étudié. On note par ailleurs que les coupes de la SEL aux différentes profondeurs sont à peu près homothétiques entre elles, le rapport des pressions de préconsolidation $\sigma_{p}^{\prime}$ représentant le rapport d'homothétie. Cette propriété d'homothétie qui est également conforme à la théorie de Cam-Clay, a, comme on le verra, des implications pratiques importantes.

On a également reproduit sur les figures 3 et 5 l'enveloppe de Mohr Coulomb de l'argile normalement consolidée. D'après tous les essais ayant été conduits jusqu'à des déformations de l'ordre de $15 \%$, il est apparu que cette enveloppe pouvait être considérée comme représentant la ligne d'état critique de l'argile de Saint-Alban. On a noté par ailleurs sur l'argile de Saint-Alban que, à un indice des vides donné, l'état critique était situé à l'intérieur de la coupe de la SEL correspondante. Cette propriété, mise en évidence sur l'argile de Saint-Alban, semble s'appliquer à toutes les argiles naturelles structurées et intactes, mais pas nécessairement aux argiles naturelles ayant subi un remaniement même très faible [12]. Elle doit donc être due à un phénomène de structuration produit par fluage, thixotropie ou cimentation, qui reste cependant à préciser.

\section{Relation entre état limite et loi effort-déformation}

Selon les principes de base des modèles Cam-Clay, une argile soumise à un état de contrainte inférieur à l'état limite correspondant à son indice des vides a un comportement parfaitement élastique, caractérisé par un module $\mathrm{E}$ et un coefficient de Poisson $\nu$. Ceci ne s'applique évidemment que dans la mesure où l'argile est supposée isotrope.

La caractérisation du comportement des argiles naturelles, anisotropes, dans le domaine surconsolidé est évidemment beaucoup plus complexe. Les études récentes sur les lois effort-déformation-temps, tendent à prouver que ce comportement, de type visco-élastique, devrait être exprimé en fonction de l'état limite plutôt qu'à partir des formulations classiques de l'élasticité. II est trop tôt pour proposer une expression mathématique globale de ce comportement mais les paramètres physiques commencent à en être connus.

Une étude détaillée [29] du fluage de l'argile de SaintAlban sous des contraintes inférieures à l'état limite a conduit d'abord au résultat présenté à la figure 6 . Les lieux des états de contraintes produisant des déformations volumétriques identiques à 1500 minutes après leur application à partir d'un état initial $\sigma_{\mathrm{vo}}^{\prime}, \mathrm{K}_{\mathrm{o}} \sigma_{\mathrm{vo}}^{\prime}$, présentent des formes manifestement reliées à celle de la coupe de la SEL au niveau étudié. Un résultat semblable est obtenu pour les déformations de cisaillement. Ainsi donc la loi effortdéformation de l'argile surconsolidée doit être exprimée non pas en fonction de la valeur absolue des contraintes appliquées mais bien en fonction de la distance à l'état limite de ces contraintes, c'est-à-dire finalement en fonc- 


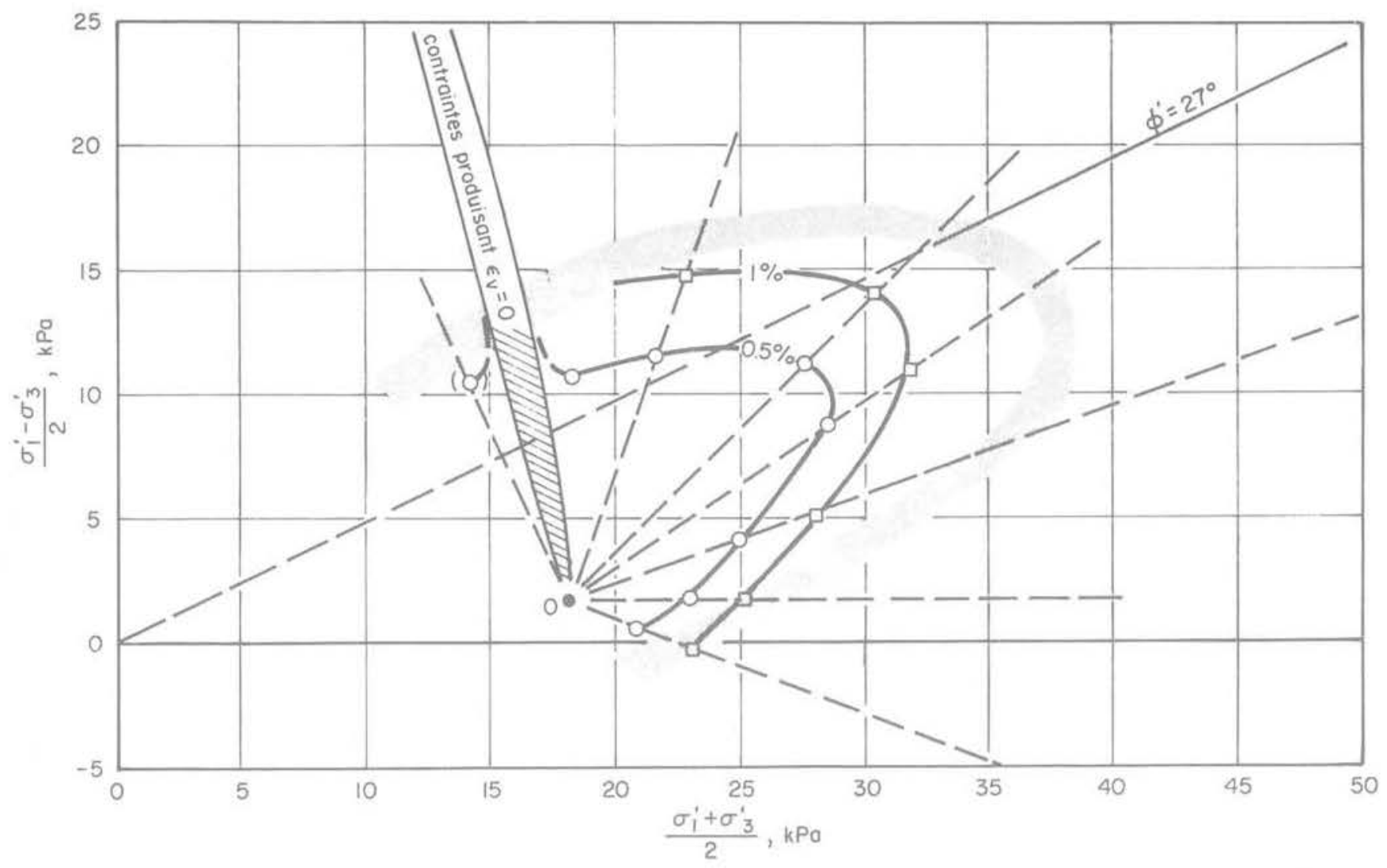

Fig. 6 Courbes d'égales déformations volumétriques de l'argile de Saint-Alban dans le domaine surconsolidé

tion de l'état limite lui-même. Une étude de laboratoire visant à préciser une telle formulation de la loi effortdéformation est actuellement en cours.

En ce qui concerne l'effet du temps sur les déformations, Tavenas et al [29] ont montré que toutes les composantes du tenseur des vitesses de déformation pouvaient s'exprimer sous la forme générale proposée par Singh et Mitchell [23] :

$$
\dot{\epsilon}=A f\left(\sigma^{*}\right)\left(\frac{t_{1}}{t}\right)^{m}
$$

dans laquelle $f\left(\sigma^{\circ}\right)$ est une fonction de contrainte, particulière pour chaque composante du tenseur des vitesses de déformation, mais exprimant dans chaque cas la distance de l'état de contrainte sous lequel $\dot{\varepsilon}$ se développe par rapport à la coupe de la SEL correspondant à l'indice des vides de l'argile étudiée. Tavenas et al [29] ont finalement suggéré qu'une formulation générale de la loi effort-déformation-temps pourrait résulter de la combinaison des notions d'état limite et d'isotaches (Suklje. [25]).

Une étude récente de l'énergie de déformation des argiles naturelles surconsolidées est venue confirmer le rôle déterminant de la SEL (Tavenas et al, 30). La figure 7 présente les courbes d'égale énergie de déformation de l'argile de Saint-Alban. Sans être parfaitement homothétiques à la SEL, ces courbes sont directement influencées par la forme de la SEL.
En conclusion, même si de nombreux points restent à préciser, tout indique que l'ensemble des propriétés de résistance, de déformation et de fluage d'une argile surconsolidée peuvent être exprimées en fonction de la SEL de cette argile. De manière plus générale ces propriétés sont directement reliées à la structure physique de l'argile c'est-à-dire à la distribution spatiale et à la résistance des contacts interparticules résultant de la déposition et de la consolidation anisotrope de l'argile, la SEL étant, comme on l'a vu l'image de cette structure dans l'espace des contraintes.

\section{Application à l'interprétation des essais}

On vient de montrer que, pour un dépôt d'argile donné, la forme de la SEL était unique, fixée par la valeur du coefficient de poussée des terres au repos $\mathrm{K}_{0}$ de l'argile en cours de consolidation, c'est-à-dire en fait par la minéralogie et la valeur résultante de l'angle de frottement effectif $\varphi^{\prime}$ de I'argile, puisque $K_{0} \simeq 0.9\left(1-\sin \varphi^{\prime}\right)$. On a également établi que, à chaque profondeur, la position et la dimension de la coupe de la SEL étaient directement fonction de la valeur locale de $\sigma_{p}^{\prime}$ et donc de l'indice des vides $e_{o}$ et que les coupes de la SEL à différentes profondeurs étaient homothétiques entre elles. La considération, de la forme elliptique particulière des SEL des argiles naturelles, et de I'homothétie des coupes de SEL à différentes profondeurs, permet d'améliorer qualitativement l'interprétation des différento essais de laboratoire et de chantier. 


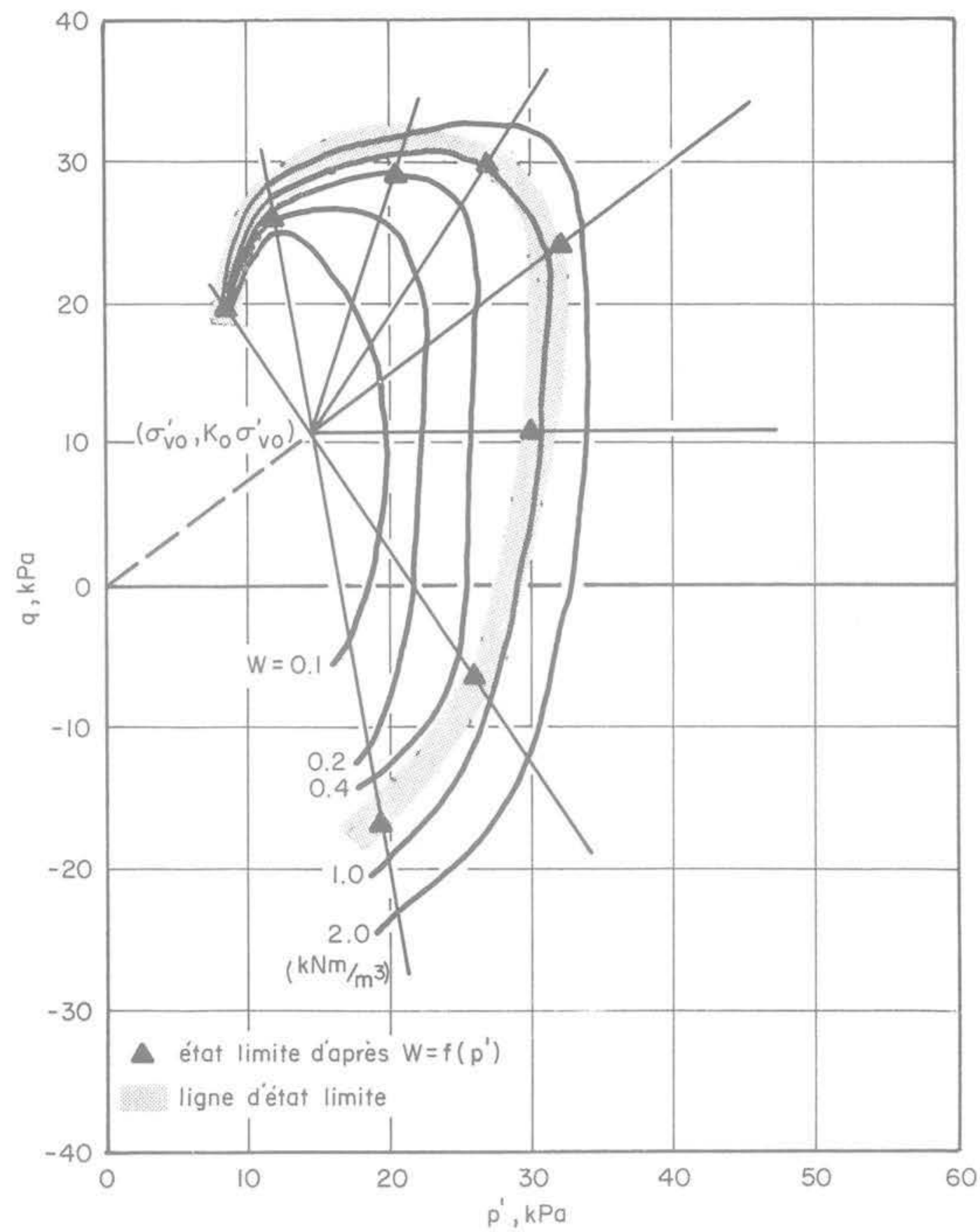

Fig. 7 Courbes d'égales énergies de déformation de l'argile de Saint-Alban (d'après Tavenas et al, [30])

Dans le cas des essais de laboratoire, chaque essai correspond à un cheminement de contraintes effectives propre. Chaque essai donnera donc un état limite en un point particulier de la SEL correspondant à ce cheminement. Connaissant la forme de la SEL des argiles naturelles, on conclut (fig. 8) qu'un essai non consolidé non drainé, réalisé à faible contrainte effective moyenne donnera une résistance au cisaillement UU plus faible qu'un essai triaxial CAU consolidé sous $\sigma_{\text {vo }}^{\prime}, K_{0} \sigma_{\text {vo }}^{\prime}$. Ainsi, contrairement à une pratique courante qui conduit à évaluer la qualité d'un essai à partir de la valeur de la résistance au cisaillement qu'il donne, il apparaît, d'une part que tout essai bien exécuté sur un échantillon d'argile surconsolidée intacte donnera un point de la SEL de cette argile, d'autre part que les différences dans les résultats obtenus par les divers essais traduisent simplement les différences dans les cheminements de contraintes effectives suivis. Par ailleurs l'existence d'une homothétie de rapport $\sigma_{p_{1}}^{\prime} \sigma_{\dot{p}_{2}}^{\prime}$ entre les coupes de la SEL à deux profondeurs (1) et (2) permet d'écrire que les états limites mesurés suivant un cheminement de contrainte donné aux deux profondeurs seront dans ce même rapport, sous réserve que les contraintes effectives en place $\sigma_{\text {vo }}^{\prime}$ soient également dans ce même rapport, c'est-à-dire que le rapport de surconsolidation soit constant dans le dépôt. En notant les cohésions respectivement UU et CAU on obtient :

$$
\frac{\sigma_{p 1}^{\prime}}{\sigma_{p 2}}=\frac{\sigma_{v o 1}^{\prime}}{\sigma_{v 02}^{\prime}}=\frac{U \dot{U}_{1}}{U U_{2}}=\frac{C A U_{1}}{C A U_{2}}=\frac{p_{i}^{\prime}}{p_{2}}
$$




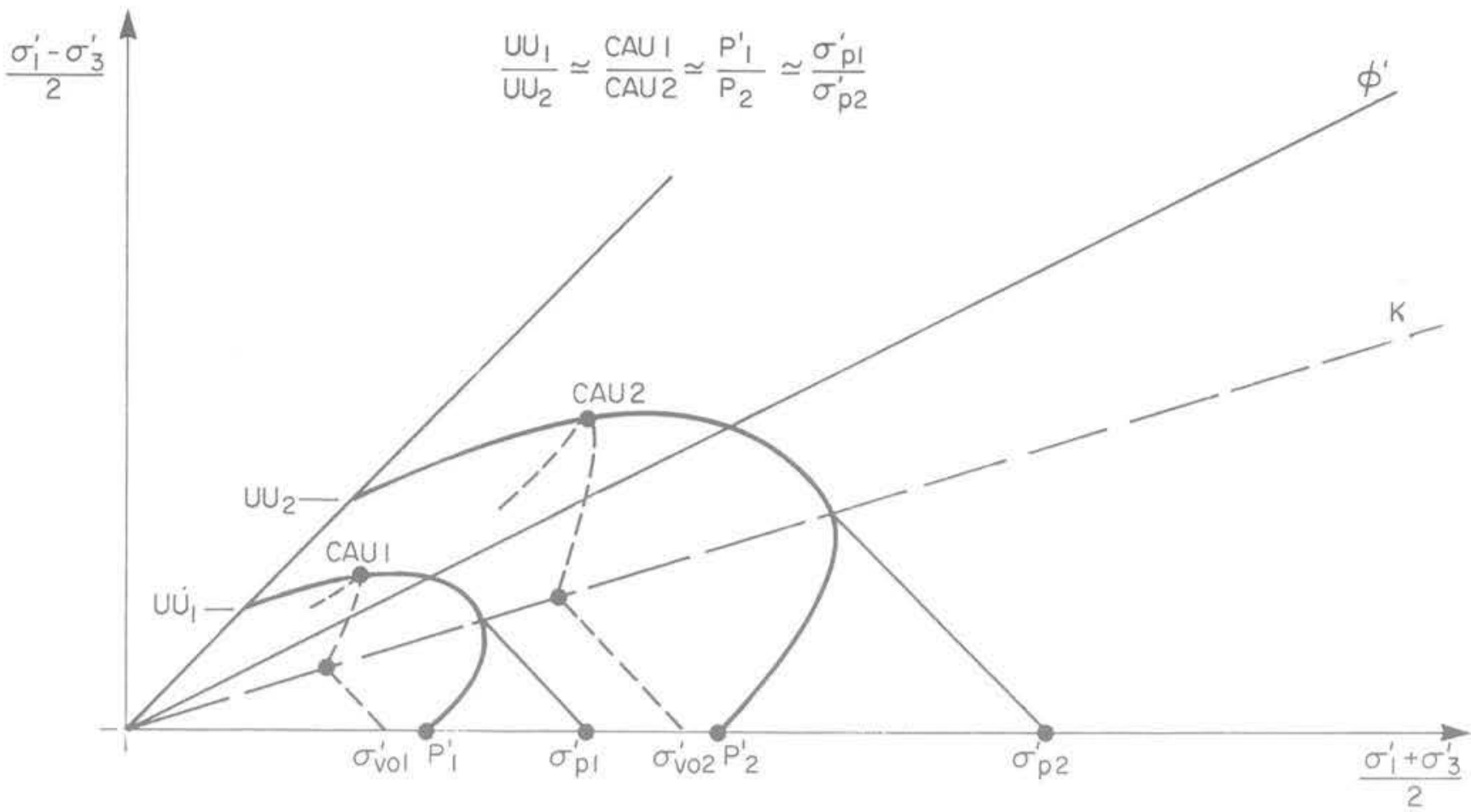

Fig. 8 SEL et interprétation des essais de laboratoire

Par combinaison, on voit ainsi qu'il y aura, dans un dépôt d'argile donné conservation des rapports UU/CAU ou encore $\mathrm{CAU} / \sigma_{\mathrm{p}}$.

Le problème est sensiblement plus complexe dans le cas des essais in situ pour lesquels on ne connait en général pas les cheminements de contraintes effectives. On sait seulement que la cohésion mesurée au scissomètre comme la résistance en pointe mesurée au pénétromètre statique ou la pression limite donnée par le pressiomètre sont toutes des représentations particulières de l'état limite de l'argile étudiée. Si on ne peut établir avec certitude le point de la SEL obtenu avec chacun de ces essais, on peut cependant appliquer à ces résultats les principes d'homothétie des SEL (fig. 9). Le fait que la résistance en pointe au pénétromètre soit représentative non seulement de la résistance (donc de l'état limite) de l'argile mais aussi de ses caractéristiques de déformation à l'état intact [21] n'empêche pas cette application dans la mesure où on a vú que les déformations étaient fonction de la SEL. On peut donc écrire

$$
\frac{C_{u 1}}{C_{u 2}}=\frac{q_{c 1}}{q_{c 2}}=\frac{\sigma_{01}^{\prime}}{\sigma_{02}^{\prime}}
$$

et, par conséquent, retrouver le principe des rapports $C_{u} / \sigma_{p}^{\prime}$ et $\mathrm{q}_{c} / \mathrm{C}_{u}$ caractéristiques de chaque dépôt. Dans le cas du pressiomètre, on dispose de quelques informations sur les cheminements de contraintes effectives depuis les essais de Baguelin et al (2) et l'étude en laboratoire de Wood et Wroth (33). Ces cheminements sont assez complexes puisque, aux variations largement étudiées des contraintes horizontales $\sigma_{2}^{\prime}$ et $\sigma_{3}^{\prime}$ s'ajoute une variation de la contrainte principale verticale $\sigma_{i}$ produite par les pressions interstitielles générées en cours d'essai. On a donc finalement un cheminement de contrainte "en spirale " dans l'espace $\sigma_{\text {; }}$ $\sigma_{2}^{\prime} \sigma_{3}^{\prime}$ (fig. 10), et qui touche l'état limite en un point encore mal défini mais correspondant à une valeur faible ou négative de $\sigma_{3}^{\prime}$. Puisque ce cheminement est totalement différent de tous les cheminements suivis dans les essais classiques, les paramètres de déformation ou de résistance obtenus au pressiomètre seront donc nécessairement différents de ceux mesurés lors des essais de laboratoire. On trouve là, la raison des échecs répétés des tentatives de comparaison entre les lois effort-déformation déterminées en laboratoire et au pressiomètre. Par contre le principe d'homothétie s'applique à la pression limite $P_{1}$ comme à tous les autres états limites et, à deux profondeurs dans un dépôt donné on aura:

$$
\frac{P_{L 1}}{P_{L 2}}=\frac{C_{u 1}}{C_{u 2}}=\frac{\sigma_{p 1}^{\prime}}{\sigma_{p 2}}
$$

Encore ici on retrouve la justification des rapports caractéristiques $q_{c} / p_{L}, p_{L} / C_{u}$ ou $p_{L} / \sigma_{p}^{\circ}$. 

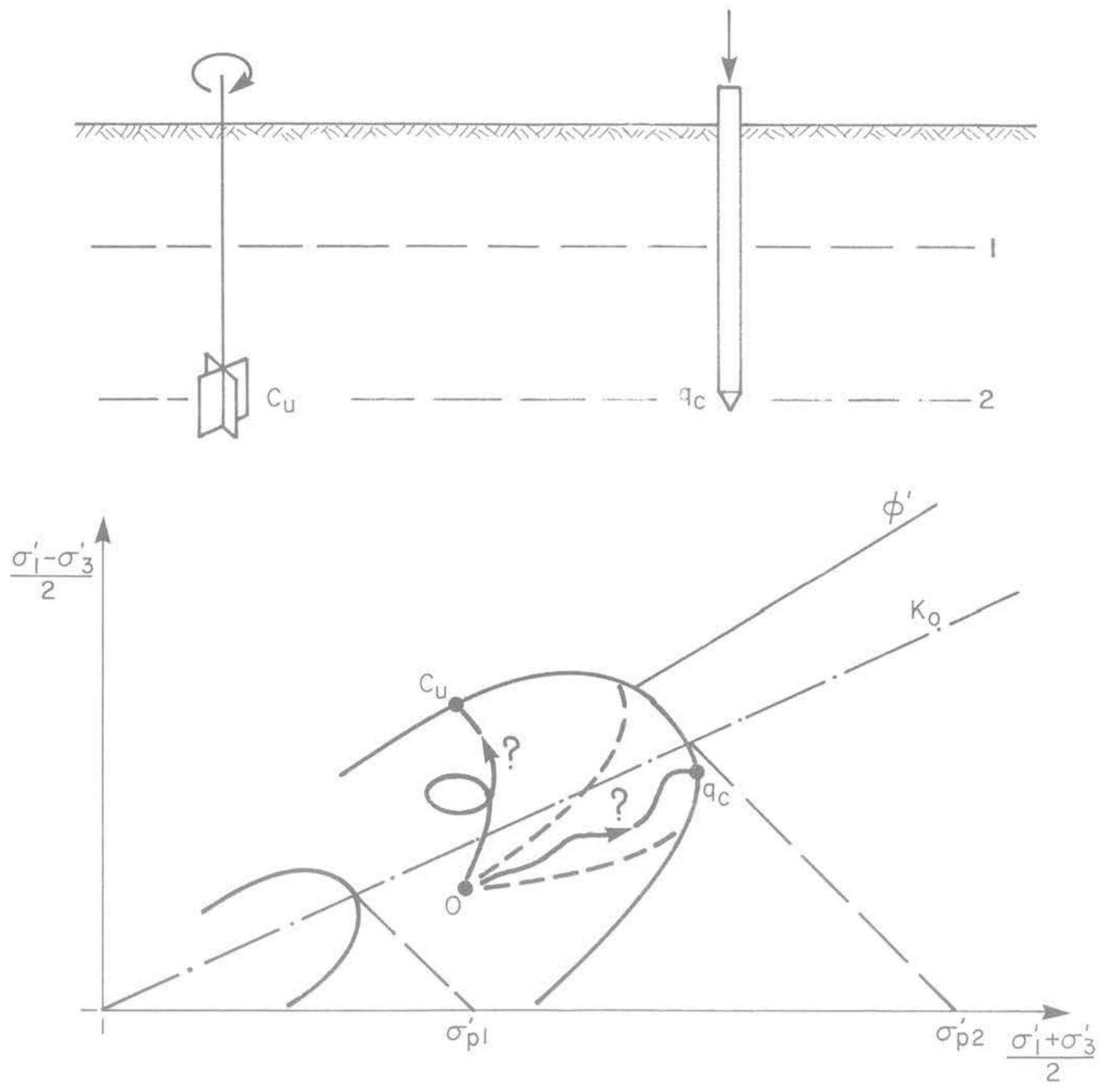

Fig. 9 SEL et interprétation des essais in situ 

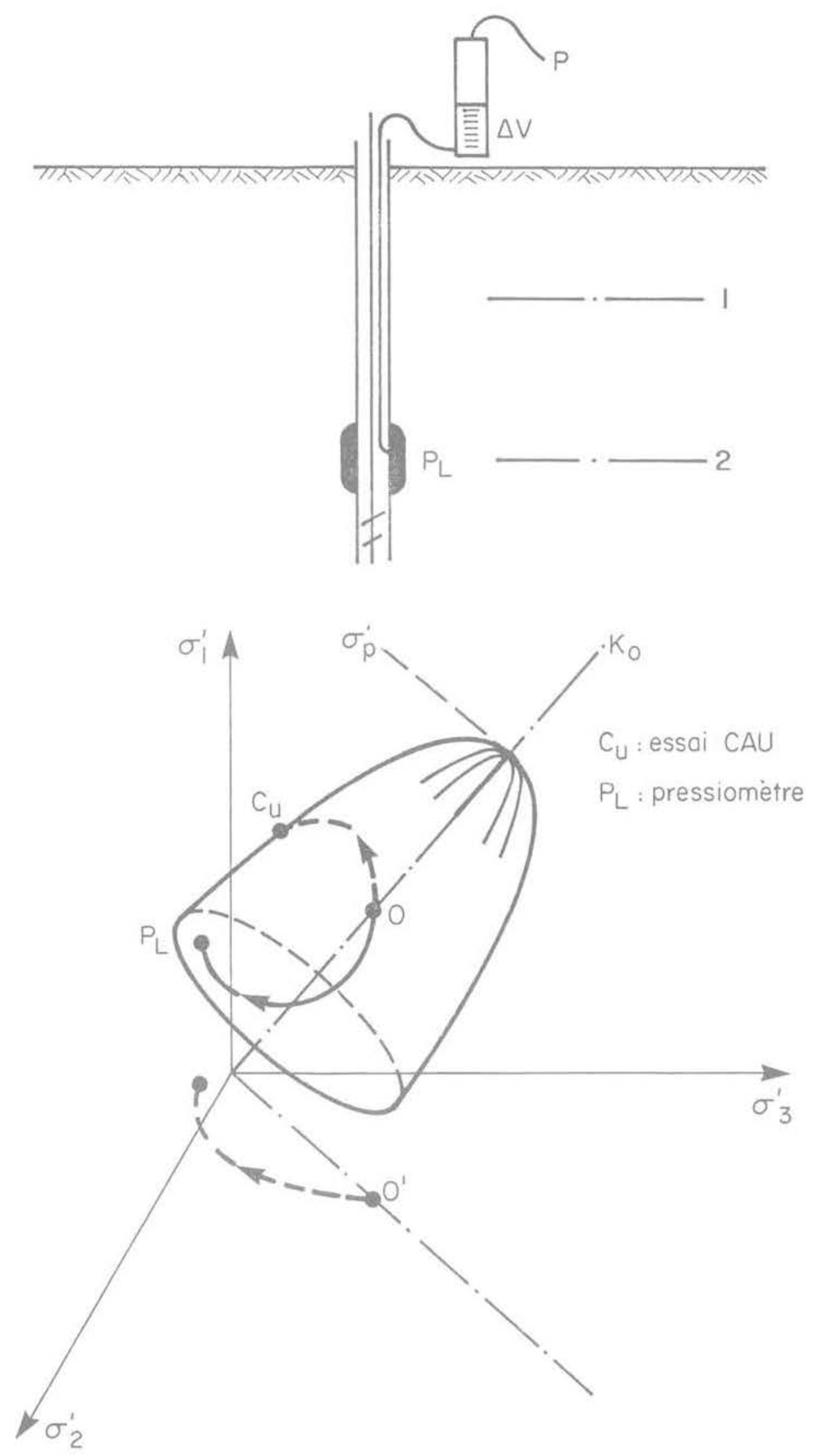

Fig. 10 SEL et interprétation de l'essai pressiométrique 


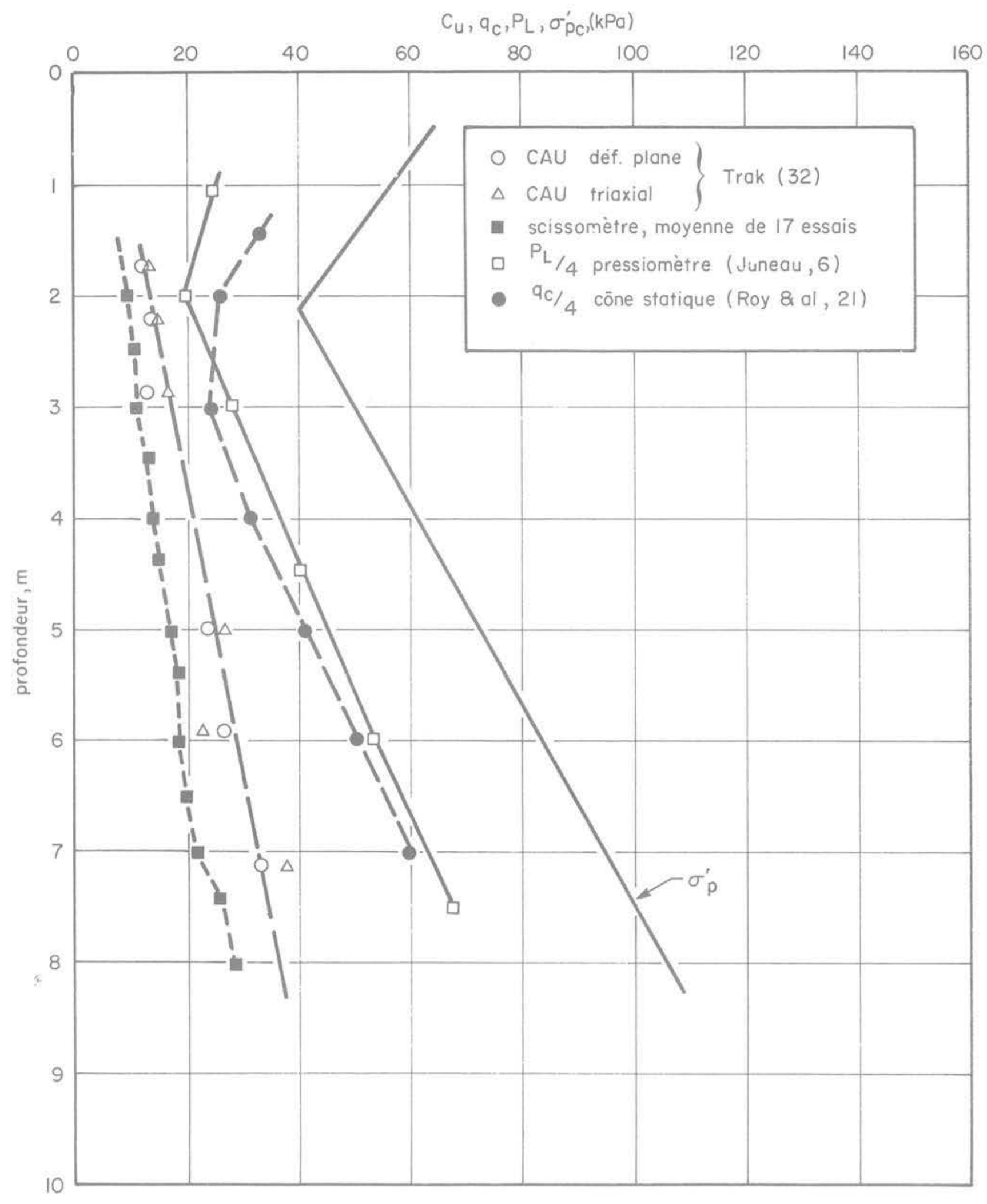

Fig. 11 Variations de $\sigma_{p^{\prime}} C_{u^{\prime}} q_{C^{\prime}}$ et $p_{L}$ en fonction de la profondeur à Saint-Alban

La figure 11, qui regroupe l'ensemble des mesures de résistance de l'argile de Saint-Alban, met bien en évidence

l'existence de rapports constants entre $\sigma_{p}^{*}, C_{u}, q_{c}$ et $p_{L}$ et confirme le principe d'homothétie des coupes de la SEL aux différentes profondeurs. 


\section{Application au calcul des ouvrages}

Les résultats présentés plus tôt indiquent clairement que, pour une argile naturelle, chaque cheminement de contraintes effectives à partir de l'état initial $\sigma_{\mathrm{vo}}^{\prime}, \mathrm{K}_{\mathrm{o}} \sigma_{\mathrm{vo}}^{\prime}$ correspond à une valeur particulière de la résistance au cisaillement ou plus généralement de l'état limite et à une forme propre de la loi effort-déformation. Ce principe s'applique également au comportement de n'importe quel ouvrage construit sur ou dans un massif d'argile. Par conséquent, si on veut arriver à une prévision convenable de tous.les aspects du comportement d'un ouvrage donné il faut d'abord identifier les cheminements de contraintes effectives suivis pendant la construction et la vie de cet ouvrage, ensuite simuler ces cheminements dans des essais de laboratoire appropriés pour en déduire la loi de comportement, et finalement établir la prévision à partir des paramètres obtenus de ces essais. Cette méthode n'est malheureusement pas ou peu applicable, car les essais classiques correspondent à des cheminements de contraintes fixés, généralement très différents de ceux suivis sous les ouvrages habituels. II est donc normal que les paramètres obtenus par ces essais ne conduisent pas à de bonnes prévisions: le cas le plus typique est celui de la résistance au cisaillement mesurée au scissomètre ou par essai CAU, qui est beaucoup plus élevée que la résistance mobilisée sous les remblais (Bjerrum, [4]). On doit cependant relever ici le cas exceptionnel de l'essai pressiométrique dont le cheminement des contraintes effectives est pratiquement identique à celui imposé au voisinage d'un pieu soumis à un chargement horizontal. Dans ce cas, comme l'ont noté Baguelin et Jezequel [1]. les paramètres de déformation et de résistance mesurés au pressiomètre correspondent bien à ceux qui caractérisent le comportement du pieu.

Dans tous les cas où une simulation des cheminements de contraintes effectives ne sera pas réalisée, on pourra tout de même arriver à une prévision correcte du comportement des ouvrages par application des principes d'homothétie et de quasi-unicité de la forme des SEL des argiles naturelles (Leroueil et Tavenas,[9]). En effet, pour un couple essaiouvrage donné, la différence entre les cheminements de contraintes et donc les valeurs correspondantes des paramètres mécaniques sera fixée et on pourra empiriquement corriger les paramètres de résistance et de déformation obtenus par essai pour les ajuster à ceux mobilisables sous l'ouvrage. On a là la justification des méthodes empiriques de calcul proposées par Ménard [14] ou par Bjerrum [4].

\section{Cas de la fouille avec soutènement ancré}

La fonction de tout soutènement (paroi moulée, berlinoise, etc.) avec ancrages précontraints est de limiter les déformations du sol, en particulier les tassements du sol à l'arrière de l'ouvrage lors de l'excavation de la fouille.

Le dimensionnement d'un tel ouvrage repose, après identification de la nature des sols et des conditions phréatiques, sur le choix du coefficient de poussée des terres $\mathrm{K}$. La limite inférieure de ce coefficient, $\mathrm{K}_{\mathrm{a}}$, implique pour être mobilisée des déplacements non négligeables du soutènement vers la fouille. Les valeurs courantes, dans le cas où on veut contrôler ces déplacements, sont supérieures à $\mathrm{K}_{\mathrm{a}}$; par exemple les normes allemandes recommandent l'usage de valeurs variant de $\left(K_{0}+K_{a}\right) / 2$ à $K_{0}$ selon la grandeur des déplacements admissibles. Quant à la détermination du coefficient de poussée des terres au repos $\mathrm{K}_{0}$, elle est associée à certaines difficultés puisqu'on connaît mal l'influence de la surconsolidation sur les contraintes horizontales: c'est ainsi qu'on prend des valeurs de $K_{0}$ plus ou moins arbitraires et comprises entre 0.5 et 1.0 .

Les notions d'état limite et de loi effort-déformation présentées plus tôt permettent une nouvelle approche de choix de K. On a vu que les déformations sont faibles à l'intérieur de la SEL, et fonctions de la distance de l'état de contraintes effectives appliqué à la SEL, et qu'elles deviennent très grandes lorsqu'on dépasse l'état limite, c'est-à-dire lorsque l'argile devient normalement consolidée. Par conséquent, pour limiter les déformations à l'arrière d'un rideau de soutènement, il suffira d'appliquer à l'aide des ancrages un état de contraintes qui soit le plus possible au centre du domaine élastique délimité par la SEL. On réalisera cette condition de la façon la plus sûre en utilisant un coefficient de poussée :

$$
K_{0}=0.9\left(1-\sin \varphi^{\prime}\right)
$$

Par contre, on voit (fig. 12) que pour une argile faiblement surconsolidée, le choix d'une valeur de $K_{0}$ élevée pourrait conduire à un passage de l'argile à l'état normalement consolidé : un tel cas a été rapporté par McRostie et al [13], où une valeur $K_{0}=1$ a conduit au développement simultané d'un déplacement du rideau vers l'intérieur du massif et d'un tassement de la surface du terrain à l'arrière du rideau.

\section{Application à l'étude des remblais sur sols mous}

De manière quasi-dogmatique, on a considéré que tous les remblais sur sols mous sont construits assez vite pour qu'on puisse distinguer d'une part la phase de construction, correspondant à un comportement non drainé de la fondation, d'autre part la phase de consolidation et donc de comportement drainé de l'argile. Toutes les méthodes de calcul de stabilité et de tassement des remblais sur sols mous sont fondées sur cette distinction, malheureusement injustifiée.

L'utilisation des notions d'état limite présentées plus tôt impose une connaissance détaillée des cheminements de contraintes effectives. Elle a donc d'abord conduit à une étude du comportement réel des fondations argileuses de remblais en cours de construction.

Une étude approfondie des pressions interstitielles générées en cours de construction sous l'axe des remblais a conduit Leroueil et al $[10,11]$ à conclure qu'une consolidation importante se développe au début de la période de construction. En effet, à ce moment là, l'argile de la fondation est en état surconsolidé et donc caractérisée par une faible compressibilité et une forte valeur de $C_{v}$. Ce phénomène, général puisqu'il a été observé sous plus de trente remblais construits sur toutes sortes de dépôts argileux, conduit à un accroissement rapide des contraintes effectives comme le montre la figure 13. Ainsi, assez rapidement après le début de la construction on atteint un état où $\sigma_{\mathrm{y}}^{\prime}=\sigma_{\mathrm{p}}^{\prime}$ et où un volume important de la fondation passe en état normalement consolidé. A ce stade, la compressibilité et donc le coefficient de consolidation de l'argile sont considérablement diminués et la poursuite de la construction se fait dans une condition pratiquement non drainée. La théorie d'état limite veut que le cheminement des contraintes effectives suive alors la coupe de la SEL à lindice des vides correspondant. Compte tenu de la forme de la SEL des argiles naturelles (fig. 5), ceci se traduit par un cheminement à $\sigma_{\mathrm{v}}^{\prime}=\sigma_{\mathrm{p}}^{\prime}=$ Cste et donc par un accroissement des pressions interstitielles

$$
\Delta u=\Delta \sigma_{i}
$$

La figure $14 \mathrm{a}$ illustre ce phénomène sous le remblai expérimental de Cubzac-les-Ponts B. Comme indiqué sur cette figure, la distance horizontale entre la ligne $\Delta \mathrm{u}=\gamma \mathrm{H}$ et la ligne expérimentale doit correspondre à $\sigma_{\mathrm{p}}^{*}-\sigma_{\mathrm{yo}}^{*}$ si l'interprétation suggérée ici est correcte. La figure $14 \mathrm{~b}$ présente la comparaison entre les valeurs de $\sigma_{p}^{\prime}$ obtenues par essai $œ$ dométrique et par interprétation de la figure $14 \mathrm{a}$ : la concordance est remarquable. Analysant plus de 90 observations sous 32 remblais, Leroueil et al [11] ont pu établir que cette concordance se retrouvait dans tous les cas d'argiles ayant un rapport de surconsolidation inférieur à 2.5 (fig. 15).

Ce passage de l'argile à l'état normalement consolidé a des conséquences très importantes sur tous les aspects du comportement des fondations de remblais en cours de construction, et donc sur toutes les méthodes de prévision de ce comportement. 

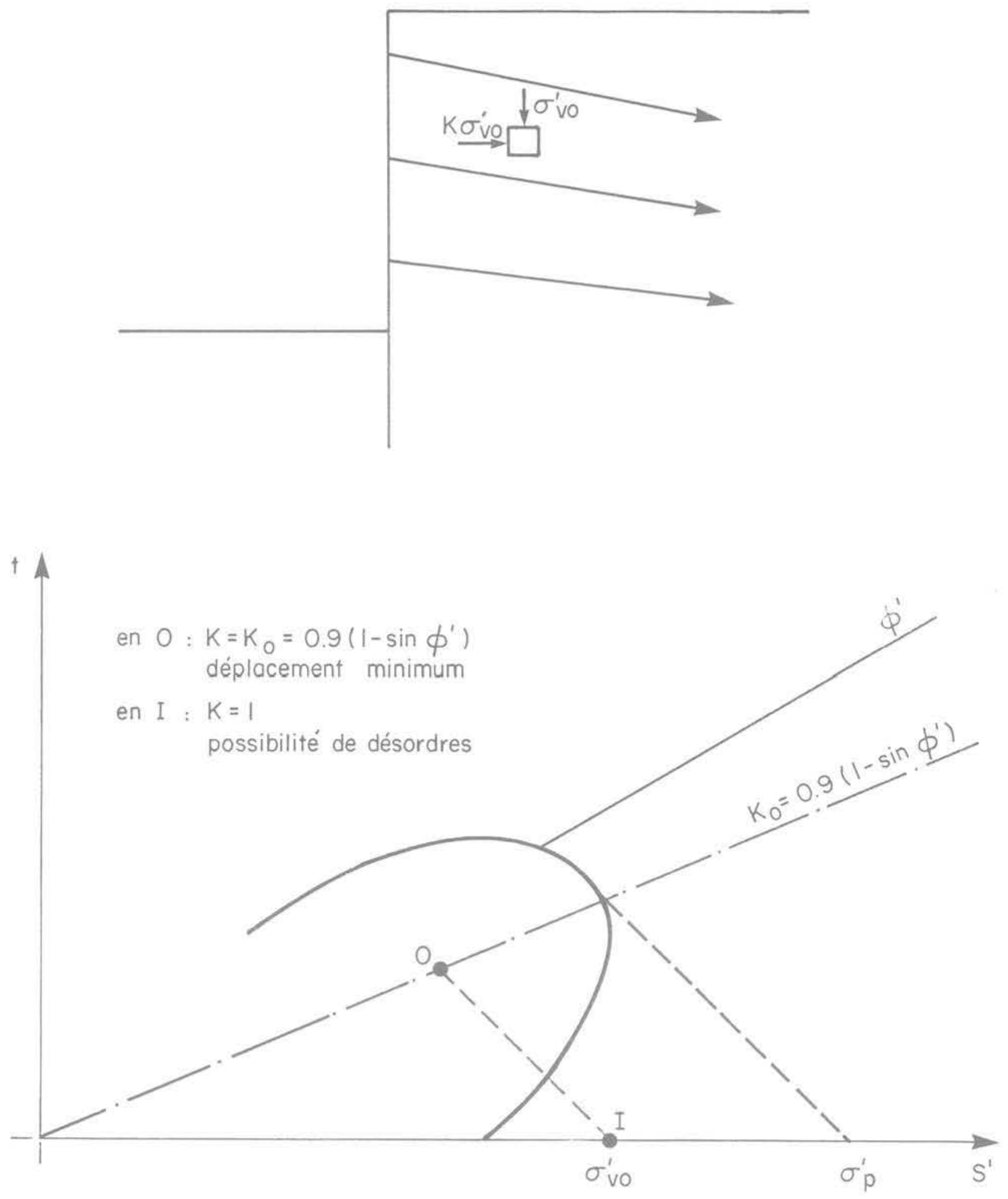

Fig. 12 SEL et le calcul des soutènements ancrés 


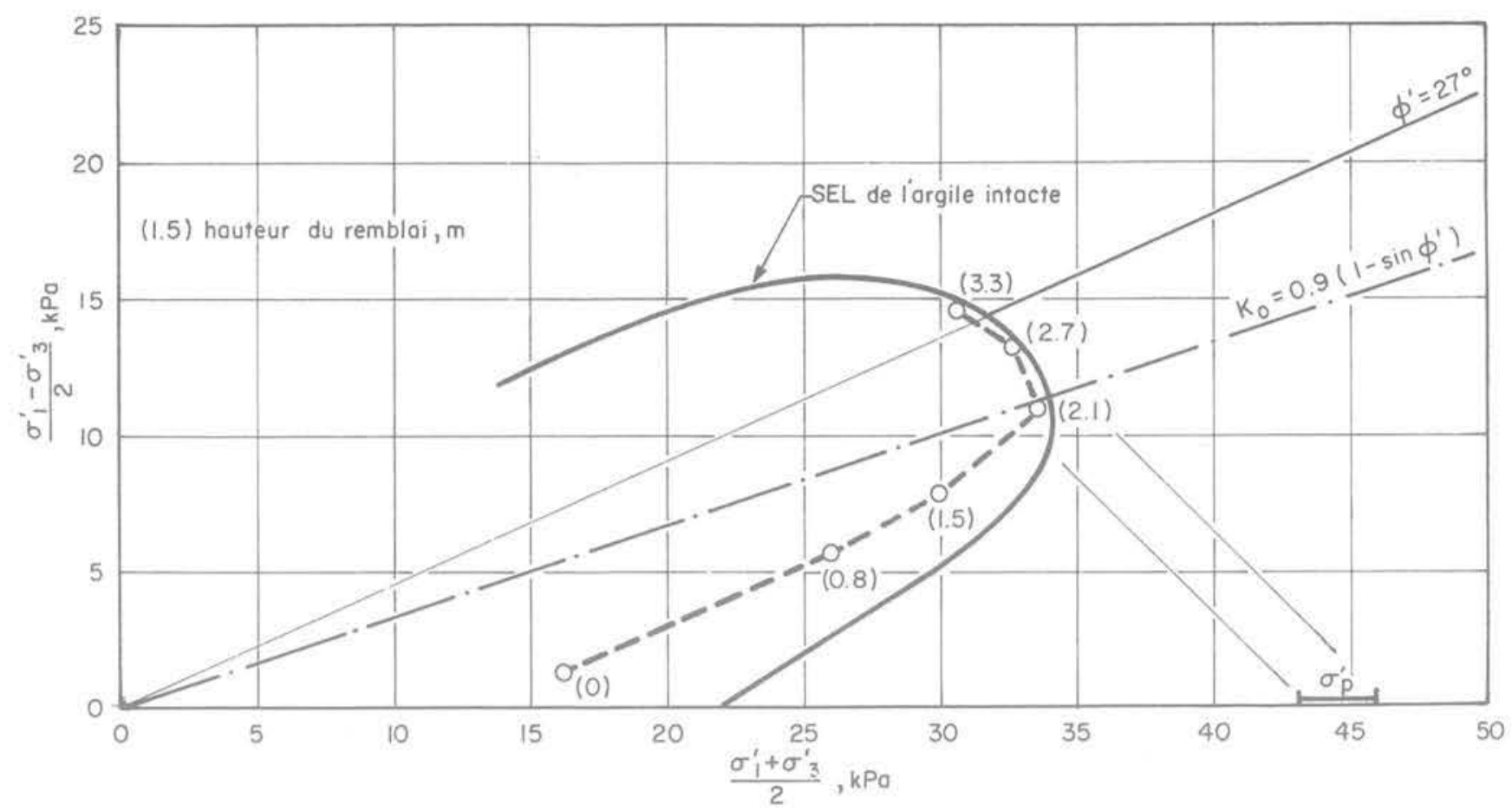

Fig. 13 Cheminement des contraintes effectives à $2.7 \mathrm{~m}$ sous l'axe du remblai C de Saint-Alban en cours de construction
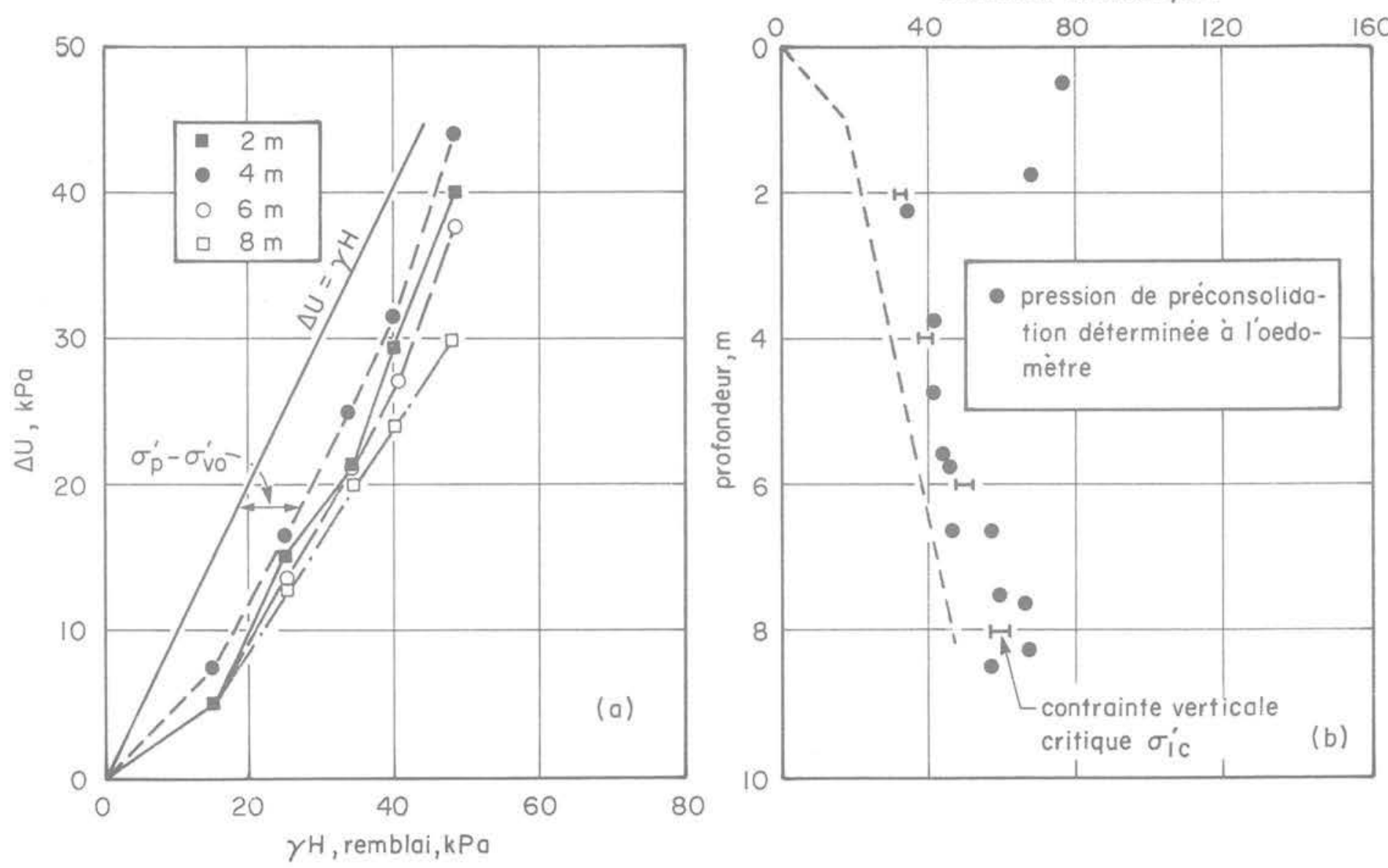

Fig. 14 Pressions interstitielles de construction sous le remblai B de Cubzac-les-Ponts 


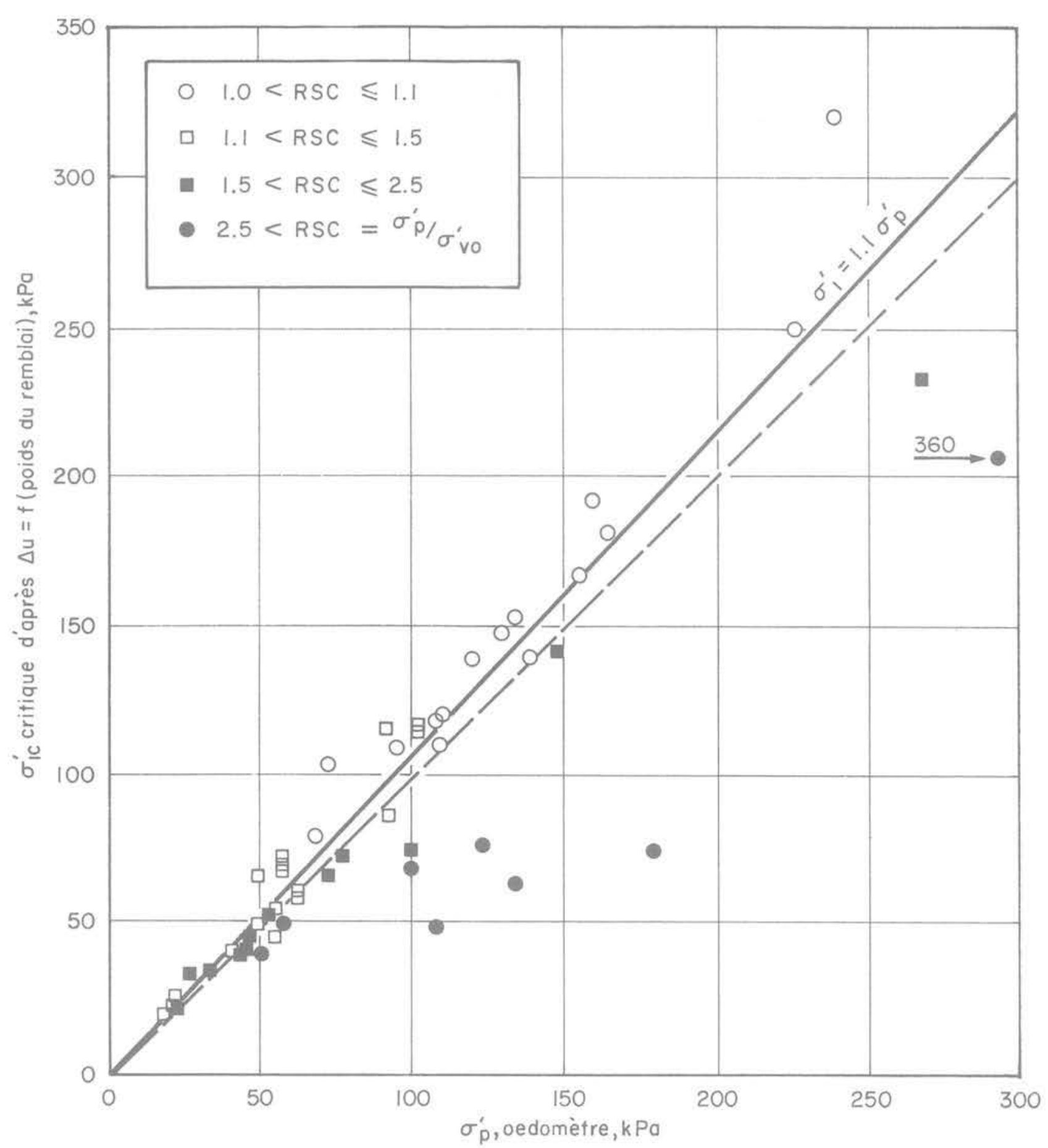

Fig. 15 Comparaison des pressions de consolidation mesurées à l'œedomètre et déduites des observations de pressions interstitielles (d'après Leroueil et al, [11]) 


\section{Tassements et déformations en cours de construction}

On a toujours admis que les tassements et les déformations latérales qui se développent dans la fondation d'un remblai en cours de construction sont le résultat de la sollicitation en état non drainé de l'argile de fondation et qu'ils doivent donc être calculés par application de la théorie de l'élasticité en supposant un coefficient de Poisson égal à 0.5 et un module d'élasticité $E_{u}$ déduit d'essais triaxiaux non drainés. Toutefois, les résultats obtenus par cette approche se sont toujours révélés de qualité douteuse (Poulos, [17]). Ceci n'est pas surprenant puisque nous venons de voir que le comportement réel de la fondation comportait initialement une phase drainée, non prise en compte dans les calculs classiques.

La figure 16 montre les cheminements de contraintes effectives suivis sous le centre d'un remblai en cours de construction et à long terme. Initialement, de $\mathrm{O}$ à $\mathrm{P}$ la fondation argileuse, en état surconsolidé, a un comportement élastique partiellement drainé. Après passage à l'état normalement consolidé en $P$. la forte réduction du coefficient de consolidation $\mathrm{C}_{\mathrm{v}}$ de l'argile se traduit par une poursuite du chargement en état non drainé et un cheminement tel que PF. Enfin, si on décidait de construire le remblai jusqu'à la rupture de sa fondation, l'argile passerait en état de plasticité en $F$ pour subir ensuite une perte de résistance jusqu'à son état critique en C. A chacune de ces trois phases de comportement, drainé, non drainé et en antiécrouissage, doit correspondre une forme particulière de la loi tassement-charge de remblais. Effectivement, on a pu observer ce phénomène lors de la construction du remblai d'essai A de Saint-Alban (La Rochelle et al, [7]). La figure 17 montre les variations du tassement sous le centre du remblai en fonction de la hauteur du remblai. On observe d'abord une augmentation faible et linéaire du tassement, correspondant au comportement élastique drainé de la fondation. En $P$, puis en $F$, où Leroueil et al [10] ont observé le passage à l'état normalement consolidé, puis le développement de la rupture et de l'anti-écrouissage de l'argile, on note deux modifications correspondantes de la loi tassement-charge. Les observations faites sous le rembiai $B$, construit deux fois plus lentement (Tavenas et al. [26]) confirment ces résultats puisque d'une part les tassements initiaux sont plus importants du fait de la durée de consolidation plus grande, d'autre part, l'accélération du tassement se produit à la hauteur de remblai où Leroueil et al [10] avaient noté le passage en état normalement consolidé.

La séquence des phases drainées puis non drainées en cours de construction affecte également le développement des déformations latérales. Dans une étude récente, Tavenas et al [31], ont mis en évidence les comportements présentés à la figure 18. Au début de la construction le déplacement latéral est beaucoup plus faible que le tassement, ce qui correspond au développement d'une consolidation importante. Puis lorsque l'argile est devenue normalement consolidée en $\mathrm{P}$, on note des accroissements identiques de $Y_{m}$ et de $S$, correspondant à une distorsion non drainée de la fondation. On voit donc que l'importance relative de la déformation latérale et du tassement en fin de construction seront fonction de la durée des phases drainées et non drainées, c'est-à-dire de la vitesse de chargement et du coefficient de consolidation de l'argile entre autres paramètres.

Pour ce qui est des déformations à long terme après la fin de construction, Leroueil et al [11] ont montré d'abord que l'amplitude du tassement à long terme devait normalement résulter d'un accroissement de la contrainte effective verticale de $\sigma_{j}$ à $\sigma_{\text {vo }}+\Delta \sigma_{v}^{\prime}$, dans l'argile normalement consolidée, c'est-à-dire suivant le cheminement de contraintes $A B C$ (fig. 16b). D'autre part, Tavenas et al [31] ont noté que les déformations latérales augmentaient linérairement en fonction des tassements, une relation

$$
\Delta Y_{m}=0.16 \Delta S
$$

étant une moyenne valable pour les remblais ayant des pentes de $1.5 / 1$ à $2 / 1$ et des facteurs de sécurité de 1.3 à
1.5. Cette variation linéaire de $Y=f(S)$ correspond à un cheminement de contraintes effectives tel que $A B$ (fig. $16 \mathrm{~b})$. Il est possible toutefois qu'une telle loi linéaire ne soit applicable que dans les premières années de la consolidation, alors qu'à plus long terme on observe un ralentissement de la déformation latérale correspondant à un rabattement du cheminement des contraintes effectives vers la ligne $\mathrm{K}_{\mathrm{o}}$ comme indiqué par la ligne $\mathrm{BC}$, (fig. 16b).

\section{Stabilité des remblais}

Dans la pratique courante, la stabilité d'un remblai en cours de construction est évaluée par une méthode de calcul en contraintes totales $(\varphi=0)$ en utilisant la résistance au cisaillement non drainé $C_{4}$ mesurée par essai au scissomètre ou par essai de laboratoire sur l'argile intacte. Pour être valable, cette méthode exige que le cheminement des contraintes effectives suivi sous le remblai lors de sa construction soit tel que Of (fig. 16a). Elle suppose de plus que la résistance mobilisable est celle de l'argile intacte, donc en un point tel que « $f$ » sur la SEL de cette argile. Or nous venons de montrer que tous les comportements observés en cours de construction correspondent à un cheminement de contrainte tel que OPFC. Le passage de la fondation à l'état normalement consolidé a des conséquences très importantes sur les caractéristiques de résistance de I'argile, ainsi que sur les cheminements de contraintes possibles, et donc sur le développement de la rupture de la fondation. En effet, Leroueil et al [12] ont montré que le passage de l'argile à l'état normalement consolidé produisait une modification de la SEL dont la partie "résistance" FY (fig. 16a) se trouvait rabaissée pour devenir, à la précision des essais près, confondue avec l'enveloppe $c_{r}^{\prime} \varphi_{\text {, }}$ correspondant aux grandes déformations et probablement à l'état critique de l'argile normalement consolidée. Par conséquent la résistance au cisaillement non drainé. mesurée par essais de laboratoire ou essais au scissomètre sur l'argile intacte, et correspondant à la zone FY de la SEL n'est certainement pas mobilisable sous un remblai puisque la SEL a pris la forme PFC avant que la fondation atteigne une condition de rupture. On retrouve là une justification d'une réduction empirique de la résistance mesurée au scissomètre pour l'évaluation de la stabilité des remblais, proposée par Bjerrum [4].

Le cheminement des contraintes effectives suivi étant tel que PFC (fig, 16a), la rupture locale de l'argile normalement consolidée s'amorce lorsque les contraintes effectives au point considéré atteignent le critère de rupture, soit en F. La plupart des argiles naturelles présentant de l'anti-écrouissage il y aura alors perte de résistance vers l'état critique $\mathrm{C}$, le cheminement de contraintes de $\mathrm{F}$ à $\mathrm{C}$ étant associé au développement de pressions interstitielles très fortes dans la fondation. Sachant cela on peut arriver à une méthode convenable des calculs de stabilité.

Lorsqu'on étudie un projet de faible envergure, pour lequel une optimisation de l'ouvrage n'est pas nécessaire, et où on peut donc rechercher simplement une valeur minimum fiable du facteur de sécurité, il suffit de considérer que tous les cheminements de contraintes effectives-dans la fondation aboutissent à l'état critique C de l'argile. On pourra donc évaluer le facteur de sécurité du remblai par référence à la résistance à l'état critique $\mathrm{C}$. Cette résistance, on l'a vu, est reliée directement à l'indice des vides et à la pression de préconsolidation de l'argile; elle a donc une valeur définie à chaque profondeur dans la fondation. On doit donc pouvoir déterminer un profil de variations de cette résistance en fonction de la profondeur et l'utiliser dans un calcul de type $\varphi=0$ comme on le fait actuellement pour le profil de résistance non drainé $C_{u}$ La question qui se pose est celle de l'évaluation de cette résistance à l'état critique. Les études en cours à l'Université Laval [32] montrent que la mesure directe par essai de laboratoire est pratiquement impossible par suite de problèmes expérimentaux (corrections de section, de membrane et de papier filtre, non homogénéité des échantillons à grande déformation). Par contre, il apparaît que la cohésion mesurée au scissomètre 


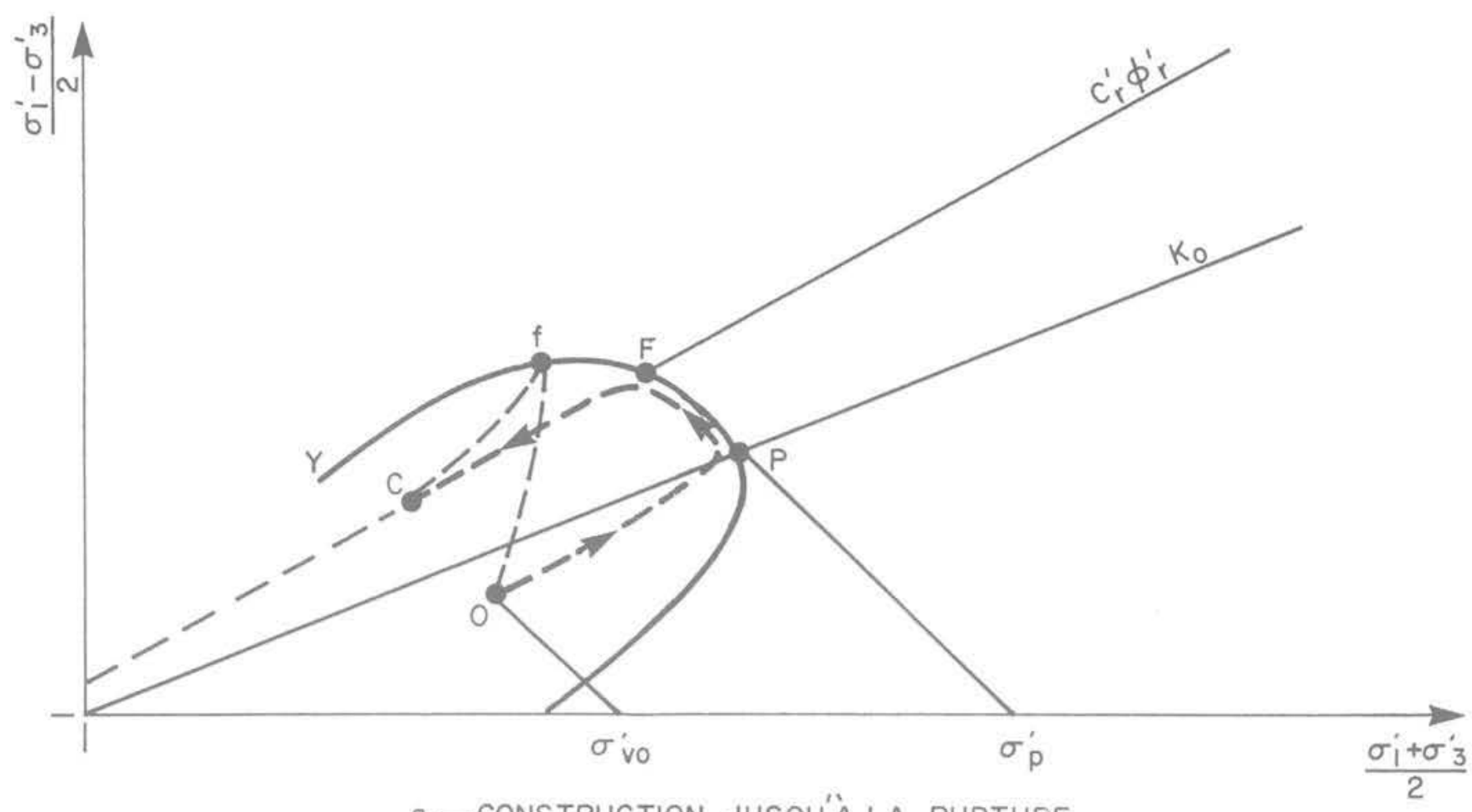

a - CONSTRUCTION JUSQU'Á LA RUPTURE

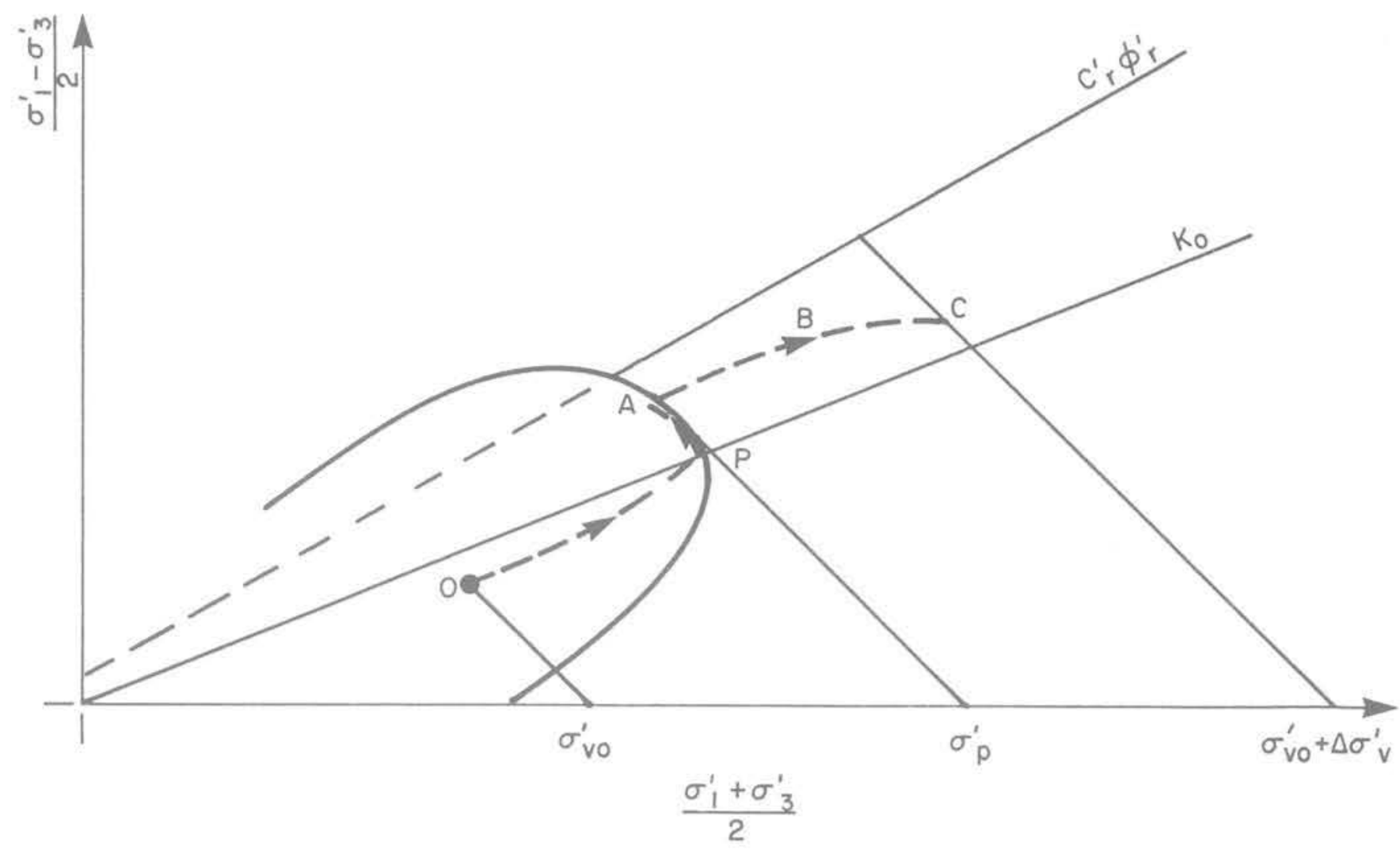

b-CONSTRUCTION SUIVIE DE CONSOLIDATION

Fig. 16 Cheminements des contraintes effectives sous le centre d'un remblai : $-a-$ conduit jusqu'à la rupture, $-b-$ en construction et en consolidation 


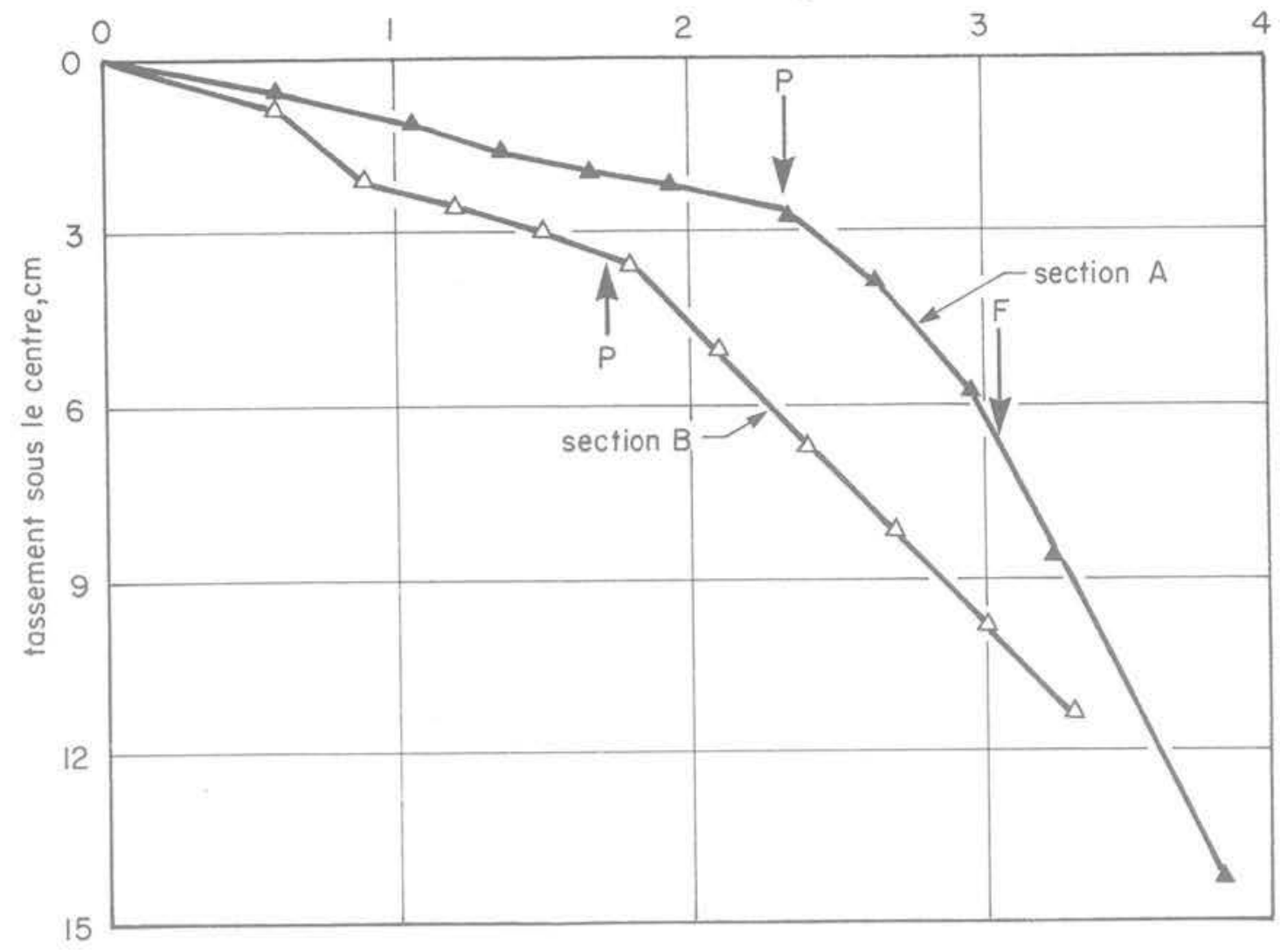

Fig. 17 Tassements de construction des remblais A et B de Saint-Alban

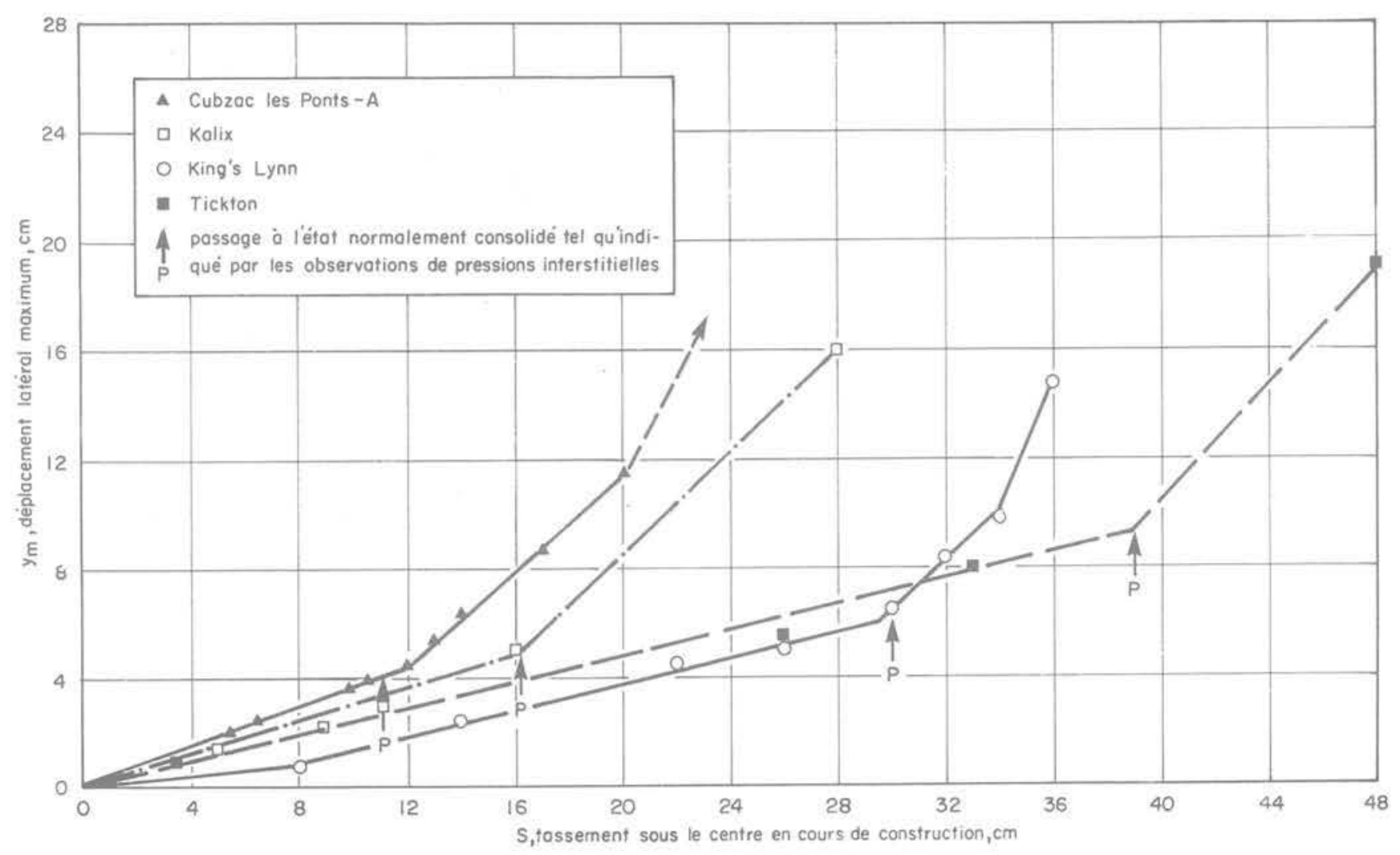

Fig. 18 Variations des déformations latérales en fonction du tassement des fondations de remblais en cours de construction (d'après Tavenas et al, [31]) 
et corrigée par application du facteur $\mu$ proposé par Bjerrum [4] donne une résistance proche de l'état critique. Par ailleurs, en montrant que la résistance disponible sous les remblais à la rupture était de la forme:

$$
\tau_{r}^{\prime}=0.22 \sigma_{p}^{\prime}
$$

Mesri [15] a probablement donné une bonne approximation de la résistance à l'état critique; une étude récente (Trak, [32]) a d'ailleurs démontré la validité d'une évaluation de la stabilité des remblais à partir de cette expression de la résistance au cisaillement de l'argile.

Si on doit au contraire optimiser le projet étudié ou s'il est nécessaire de recourir à la technique de la construction par étape, il devient essentiel de pouvoir suivre les variations du facteur de sécurité en cours de construction. Ceci ne pourra se faire qu'en utilisant une analyse en contraintes effectives prenant en compte les pressions interstitielles observées dans la fondation qui aura été préalablement instrumentée. Le cheminement des contraintes effectives suivi à l'approche de la rupture étant tel que PFC (fig. 16a), les paramètres de résistance à prendre en compte seront ceux correspondant à FC, c'est-à-dire les paramètres $C_{t}^{\prime}, \varphi_{\text {, }}^{\prime}$ mesurés à grandes déformations dans l'argile normalement consolidée. L'utilisation d'une telle méthode d'analyse permettra de suivre exactement les variations des contraintes effectives et des résistances disponibles correspondantes dans la fondation, et donc d'obtenir une image plus réelle des variations du facteur de sécurité. En effet, tant quaucun point de la fondation n'aura atteint la condition de rupture locale (F), la résistance disponible sera celle en F. Cette résistance étant nettement plus élevée que la résistance à l'état critique $C$, il s'ensuit que les facteurs de sécurité calculés seront supérieurs à ceux obtenus par l'analyse de type $\varphi=0$. La figure 19 illustre cette différence et met en évidence tout le bénéfice que I'on peut tirer de l'utilisation de l'analyse en contraintes effectives. Tavenas et al [28] ont d'ailleurs décrit un cas d'application de cette méthode à l'étude de sept remblais dans la vallée du Saint-Laurent, Québec, où une accélération du rythme de construction et une śconomie substantielle dans le coût du projet ont pu être obtenues. II convient cependant de faire attention aux conséquences du développement d'un anti-écrouissage dans l'argile de fondation. En effet, dès qu'un élément de la fondation passe à la rupture locale en $\mathrm{F}$, le cheminement de contraintes effectives est imposé suivant FC et la résistance au cisaillement disponible localement diminue donc vers la résistance à l'état critique au fur et à mesure de l'augmentation des pressions interstitielles due à l'antiécrouissage. De ce fait, à partir du développement de la rupture locale en un point de la fondation, le facteur de sécurité calculé par une analyse en contraintes effectives va subir une diminution rapide vers la valeur de 1.0 , correspondant à la résistance à l'état critique, obtenue au moment de la rupture totale de la fondation. Ainsi, c'est seulement lors de la rupture totale, et sous réserve d'une mesure convenable des pressions interstitielles à ce moment, que les deux analyses en contraintes effectives et en contraintes totales donneront le même facteur de sécurité de 1.0. Pour le projeteur désirant faire usage du bénéfice possible par application du calcul en contraintes effectives, le seul impératif est de s'assurer qu'aucun point de la fondation ne passe en état de rupture locale, c'est-à-dire que le facteur de sécurité ne se situe pas dans sa phase de décroissance rapide. Selon les observations faites par Trak [32] à Saint-Alban it semble que cette condition soit satisfaite si on maintient le facteur de sécurité à des valeurs supérieures à 1.3. Les observations

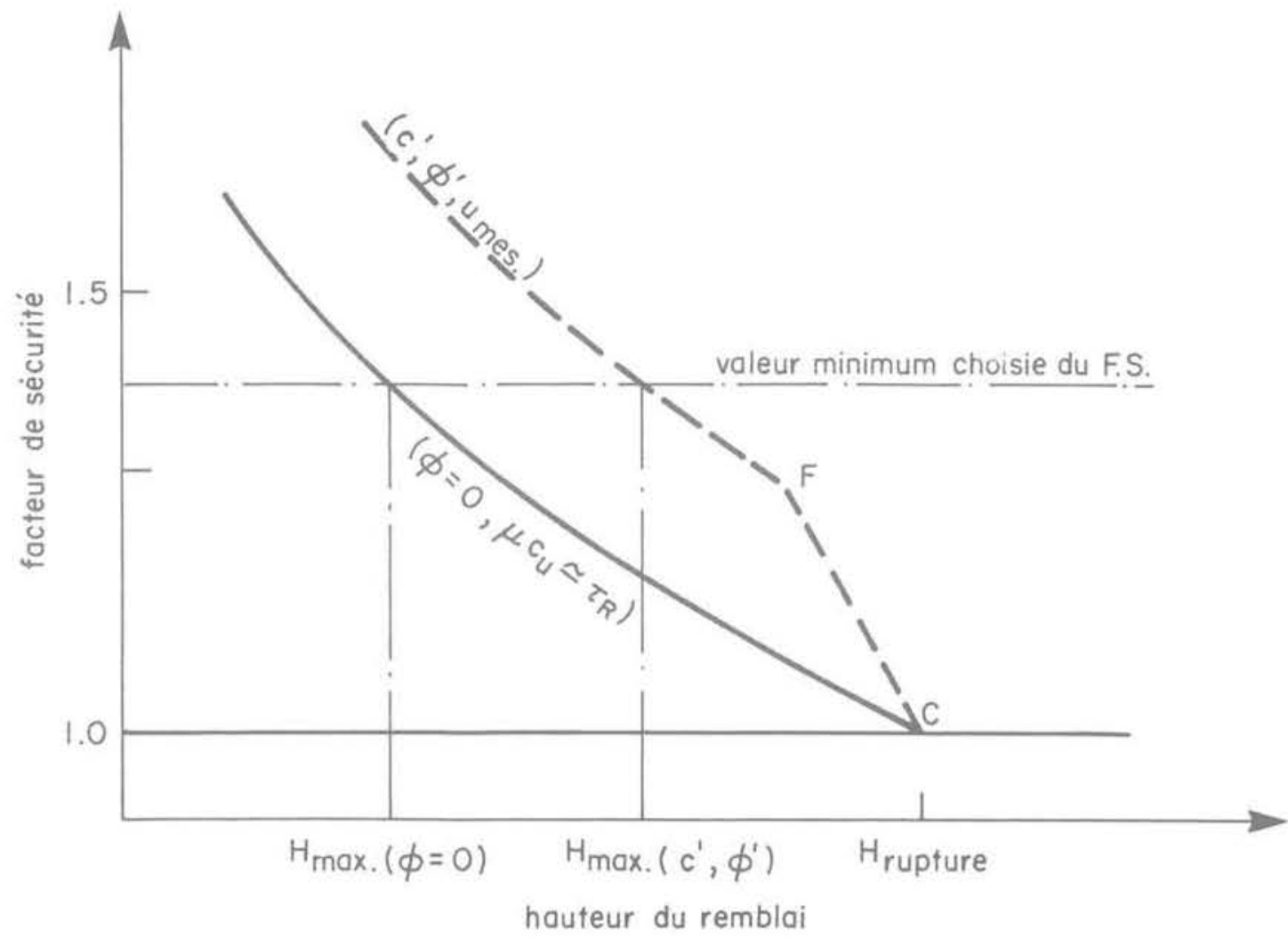

Fig. 19 Variations des facteurs de sécurité calculés en contraintes totales en fonction de la hauteur de remblai (d'après Tavenas et al, [28]) 
des pressions interstitielles sous le centre du remblai fournissent un moyen de contrôle supplémentaire ; en effet, le développement d'une condition de rupture locale et d'anti-écrouissage sera mis en évidence par des accroissements de pressions interstitielles $\Delta u$ nettement supérieurs à l'accroissement de charge du remblai $\Delta \gamma \mathrm{H}$.

On voit donc que la mise en œuvre des concepts d'état limite et d'état critique, en imposant une étude détaillée des cheminements de contraintes effectives suivis dans les fondations de remblais, a permis de développer une meilleure compréhension du comportement de ces fondations et, partant, de renouveler les méthodes d'analyse de ces ouvrages.

\section{Conclusion}

Les études expérimentales réalisées récemment sur des argiles molles d'origines diverses ont montré que les concepts d'état limite et d'état critique, s'appliquent bien, de manière qualitative, à la description du comportement mécanique des argiles naturelles. Si les modèles mathématiques Cam-Clay et Cam-Clay modifié, fondés sur ces concepts, ne peuvent s'appliquer correctement aux argiles naturelles parce qu'ils en négligent les caractéristiques d'anisotropie et de viscosité, il reste que les lois rhéologiques de ces matériaux sont certainement gouvernées par les caractéristiques d'état limite et qu'on devrait pouvoir prochainement proposer des formulations assez simples et générales de ces lois.

L'utilisation des notions d'état limite et d'état critique conduit par ailleurs à une approche nouvelle, intégrée, et relativement simple des problèmes courants de la mécanique des argiles. Essentiellement on a vu:

1 Qu'elle met en évidence la nécessité absolue de définir et d'étudier les cheminements des contraintes effectives dans l'analyse de tout problème de comportement d'argile.

2 Qu'elle permet une meilleure évaluation de la qualité des essais de laboratoire et une utilisation plus juste des caractéristiques mécaniques obtenues de ces essais.

3 Qu'elle conduit à la compréhension des limitations des essais in situ et à la justification des méthodes empiriques d'interprétation et d'utilisation des résultats de ces essais.

4 Qu'elle a imposé une remise en question des méthodes classiques d'analyse de la stabilité et des déformations des remblais sur sols mous. Elle a conduit, après l'examen des cheminements de contraintes effectives réellement suivis sous les remblais en cours de construction et à long terme, à la proposition d'une nouvelle approche des calculs de tassement, de déformation latérale et de stabilité, souvent plus simple que l'approche antérieure, et certainement plus fiable.

\section{Remerciements}

Les travaux présentés ici ont été développés à l'Université Laval depuis 1970 avec la collaboration active de Madame Fauveau-Brucy et Messieurs M. Brucy, C. Chapeau, J.-P. Des Rosiers, R. Juneau, D. Michaud, J. Sarrailh, B. Trak, Vu, K.M. étudiants gradués et de Messieurs J.-P. Dussault, J.-Y. Julien, S. Paré, et M. Pouliot, techniciens.

Les auteurs remercient leurs collègues $P$. La Rochelle et M. Roy, professeurs à I'Université Laval, G. Pilot, J.-P. Magnan, C. Mieussens, M. Peignaud et D. Queyroi des Laboratoires des Ponts et Chaussées de Paris, Toulouse, Angers et Bordeaux, et R. Garneau de Terratech Ltée, Montréal pour leur participation active à la recherche. leurs discussions et commentaires.

Ces travaux ont été financés par des octrois du Ministère de l'Éducation du Québec, du Conseil National de Recherches du Canada, et par un accord de coopération FranceQuébec.

\section{Références Bibliographiques}

[1] BAGUELIN F., et JEZEQUEL J.-F. - Étude expérimentale du comportement de pieux sollicités horizontalement. Annales de IITBTP, Supplément au $n^{\circ}$ 297, série SF/1, pp. 153-204, 1972.

[2] BAGUELIN F., JEZEQUEL J.-F. et LE MÉHAUTÉ A. - Étude des pressions interstitielles développées lors de l'essai pressiométrique. C.R. $8^{\circ}$ conf. int. Mec. Sols \& Trav. Fond., Vol. 1.1, pp. 19-24, Moscou, 1973

[3] BJERRUM L. - Engineering Geology of Norwegian normally consolidated marine clays as related to settlements of buildings. Géotechnique. Vol. 17 (2), pp. 81-118, 1967.

[4] BJERRUM L. - Problems of soil mechanics and construction on soft clays. Rapport sur l'état des connaissances, session 4 , C.R. $8^{\circ}$ Conf, int. Mec. Sols \& Trav. Fond., Vol. 3, pp. 111-159, Moscou, 1973.

[5] CROOKS J.H.A, et GRAHAM J. - Geotechnical properties of the Belfast estuarine deposits. Géotechnique, Vol. 26 (2). pp. 293-315, 1976.

[6] JUNEAU R. - Contribution au développement de la mise en place de la sonde pressiométrique dans les argiles sensibles. Thèse de maîtrise. Département de Génie Civil, Université Laval, Québec, 1975

[7] LA ROCHELLE P., TRAK B., TAVENAS F, et ROY M. - Failure of a test embankment on a sensitive Champlain clay deposit. Rev. Can. de Géot. Vol. 11 (1), pp. 142-164, 1974.

[8] LEROUEIL S. - Quelques considérations sur le comportement des argiles sensibles. Thèse de doctorat, Dépt. Génie civil, Université Laval, Québec, 1977.

[9] LEROUEIL S. et RAVENAS F. - Discussion de Crooks J.H.A. and Graham J. Géotechnique, Vol, 27 (3), pp. 441-446, 1977.

[10] LEROUEIL S., TAVENAS F., TRAK B., LA ROCHELLE $P$. et ROY M. - Construction pore pressures in clay foundations under embankments, Part 1: The Saint-Alban test fills. Rev. Can. de Géot., Vol. 15 (1). pp. 54-65, 1978.

[11] LEROUEIL S., TAVENAS $F_{\text {. }}$ MIEUSSENS $C$. et PEIGNAUD M. - Construction pore pressures in clay foundations under embankments, Part II: generalized behaviour. Rev. Can, de Géot, Vol. 15 (1), pp. 66-82, 1978.

[12] LEROUEIL S., TAVENAS F., BRUCY F., LA ROCHELLE P, et ROY M. - Effect of destructuration on the behaviour of natural clays. Soumis pour publication. ASCE Journ. Géot. Eng. Div., 1978.

[13] MC ROSTIE G.C., BURN K.-N. et MITCHELL R.-J. - The performance of tied-back sheet piling in clay. Rev. Can, de Géot. Vol. 9 (2), pp. 206-218, 1972.

[14] MÉNARD L. - Règles pour le calcul de la force portante et du tassement des fondations en fonction des résultats pressiométriques. C.R. $6^{\mathbf{e}}$ Conf. Int. Mec. Sols \& Trav. Fond., Vol. II. pp. 295-299, Montréal, 1965.

[15] MESRI G. - Discussion de Ladd et Foott. ASCE Journ. Géot, Eng. Div, Vol. 101 (GT4), pp. 409-412, 1975

[16] OHTA H., et HATA S. - On the state surface of anisotropically consolidated clays. Proc. Jap. Soc. Civ. Eng., n 196 . pp. 117-124, 1971.

[17] POULOS H.-G. - Difficulties in prediction of horizontal deformations of foundations. ASCE, Journ. Soil. Mech. \& Found. Div., Vol. 98 (SM8), pp. 843-848, 1972

[18] ROSCOE K.-H., SCHOFIELD A.N. et WROTH, C.-P. - On the yielding of soils, Géotechnique, Vol. 8 (1), pp. 22-53, 1958

[19] ROSCOE K.-H., SCHOFIELD A.-N. et THURAIRAJAH. A. Yielding of clays in states wetter than critical. Géotechnique, Vol. 13 (3), pp. 211-240, 1963.

[20] ROSCOE K.-H. et BURLAND J.-B. - On the generalized stress-strain behaviour of wet clay. Engineering plasticity (Éd. J. Heyman, F.A. Leckie), Gambridge University Press, pp. 535-609, 1968.

[21] ROY M., MICHAUD D., TAVENAS F., LEROUEIL S., LA ROCHELLE P. - The interpretation of static cone penetration tests in sensitive clays. C.R. ESOPT. Vol. 2.2. pp. 323-331. Stockholm, 1974.

[22] SARRAILH J. - Contribution à l'étude des méthodes d'échantillonnage des argiles sensibles. Thèse de maîtrise. Dépt. Génie civil, Université Laval, Québec, 1975.

[23] SINGH A.-W., MITCHELL J.-K. - General stress-strain-time function for soils. ASCE Journ. Soil Mech. and Found. Div., Vol. 94 (SM1), pp. $21-46,1968$. 
[24] SKEMPTON A.-W. - Vane tests in the alluvial plain of the River Forth near Grangemouth. Géotechnique, Vol. I, pp. 111124, 1948.

[25] SUKLJE L. - The analysis of the consolidation process by the isotaches method. C.R. $4^{\circ}$ conf. int. Mec. Sols \& Trav. Fond. Vol. 1, pp. 200-206, Londres, 1957.

[26] TAVENAS F., CHAPEAU C., LA ROCHELLE P. et ROY M. Immediate settlements of three test embankments on Champlain clay. Rev. Can. de Géot. Vol. 11 (1), pp. 109-141, 1974.

[27] TAVENAS F., LEROUEIL S. - Effects of stresses and time on yielding of clays. C.R. $9^{\circ}$ Conf. int. Mec. Sols et Trav. Fond., Vol. 1. pp. 319-326, Tokyo, 1977.

[28] TAVENAS F., BLANCHET R., GARNEAU R., LEROUEIL S. The stability of stage constructed embankments on Champlain clay. Rev. Can. de Géot., Vol. 15 (2).
[29] TAVENAS F., LEROUEIL S., LA ROCHELLE P., ROY M. Creep behaviour of an undisturbed lightly overconsolidated clay. Rev. Can. de Géot. Vol. 15, pp. 402-423, 1978.

[30] TAVENAS F., DES ROSIERS J.-P., LEROUEIL S., LA ROCHELLE P., ROY M. - The use of strain energy as a yield and creep criterion for soft clays. Soumis pour publication, Géotechnique, 1978.

[31] TAVENAS F., MIEUSSENS C. BOURGES F. - Lateral displacements in clay foundations under embankments. Soumis pour publication, ASCE Géot. Eng. Div., 1978.

[32] TRAK B. - Contribution à l'étude de la stabilité des remblais sur sols mous. Thèse de doctorat à être présentée à l'École des Gradués de I'Université Laval, Québec, 1978.

[33] WOOD D.-M., WROTH C.-P. - Some laboratory experiments related to the results of pressuremeter tests. Géotechnique, Vol. 27 (2), pp. 181-201, 1977. 
